TRANSACTIONS OF THE

AMERICAN MATHEMATICAL SOCIETY

Volume 360, Number 12, December 2008, Pages 6197-6230

S 0002-9947(08)04366-3

Article electronically published on July 28, 2008

\title{
FLUCTUATION OF A PLANAR BROWNIAN LOOP CAPTURING A LARGE AREA
}

\author{
ALAN HAMMOND AND YUVAL PERES
}

\begin{abstract}
We consider a planar Brownian loop $B$ that is run for a time $T$ and conditioned on the event that its range encloses the unusually high area of $\pi T^{2}$, with $T \in(0, \infty)$ being large. The conditioned process, denoted by $X$, was proposed by Senya Shlosman as a model for the fluctuation of a phase boundary. We study the deviation of the range of $X$ from a circle of radius $T$. This deviation is measured by the inradius $\mathrm{R}_{\mathrm{in}}(X)$ and outradius $\mathrm{R}_{\text {out }}(X)$, which are the maximal radius of a disk enclosed by the range of $X$, and the minimal radius of a disk that contains this range. We prove that, in a typical realization of the conditioned measure, each of these quantities differs from $T$ by at most $T^{2 / 3+\epsilon}$.
\end{abstract}

\section{INTRODUCTION}

The goal of this paper is to analyse the fluctuations of a planar Brownian loop under the condition that it encircles a large area. Throughout, $B:[0, T] \rightarrow \mathbb{R}^{2}$ will denote a standard planar Brownian loop, that is, a planar Brownian motion with initial location $B(0)=0$ that is conditioned on the event that $B(T)=0$. (Equivalently, $B$ has the law of $W(t)-\frac{t}{T} W(T)$, where $W:[0, T] \rightarrow \mathbb{R}^{2}$ is a planar Brownian motion with $W(0)=0$.) Let $\operatorname{enc}(B)$ denote the closure of the union of all bounded components of $\mathbb{R}^{2} \backslash\{B(t): t \in[0, T]\}$. Then our conditioning takes the form

$$
|\operatorname{enc}(B)| \geq \pi T^{2}
$$

where $|\cdot|$ denotes two-dimensional Lebesgue measure. Note that by the spatialtemporal scaling satisfied by Brownian motion, the law of the conditioned process is the same as that obtained from sampling a Brownian loop run for a unit of time that is conditioned to enclose an area exceeding $\pi T$, and then dilating space by a factor of $\sqrt{T}$. Throughout, we will define the process on the interval $[0, T]$. The conditioned process will be denoted by $X:[0, T] \rightarrow \mathbb{R}^{2}$. As we discuss in Section 2 , a classical variational principle suggests that the range $X[0, T]$ takes a form close to that of a circle of radius $T$. The principal aim of this paper is to investigate the magnitude of the deviation of the range $X[0, T]$ from such a circle. Our main theorem provides a bound on a quantity that measures this deviation. To be precise, for a planar compact set $K$, the inradius $\mathrm{R}_{\text {in }}(K)$ of $K$ is the maximal radius of a circle lying in $K$, while the outradius $\mathrm{R}_{\text {out }}(K)$ is the minimal radius of any circle

Received by the editors February 3, 2006 and, in revised form, June 3, 2006.

2000 Mathematics Subject Classification. Primary 60J65; Secondary 60F10.

The research of the second author was supported in part by NSF grants \#DMS-0244479 and \#DMS-0104073. 


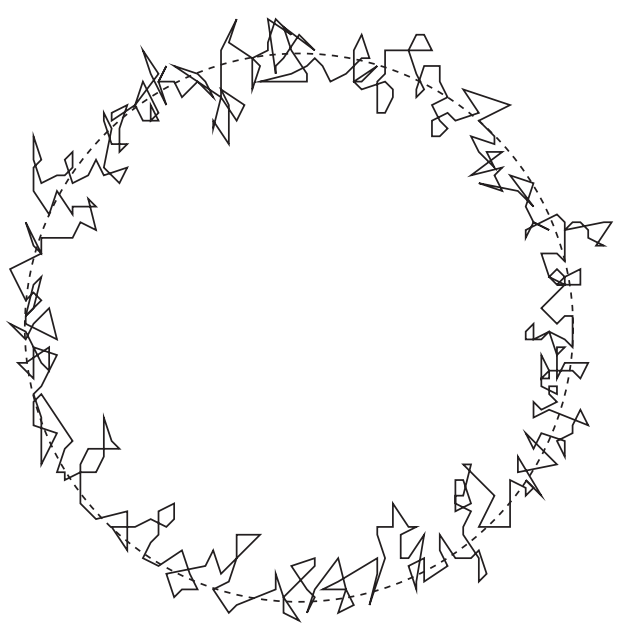

FiguRE 1. A sketch of the conditioned motion.

in which $K$ is contained. We will write $\mathrm{R}_{\text {in }}(B)$ and $\mathrm{R}_{\text {out }}(B)$ for $\mathrm{R}_{\text {in }}(\operatorname{enc}(B))$ and $\mathrm{R}_{\text {out }}(\operatorname{enc}(B))$, adopting the same shorthand for the process $X$.

Theorem 1. Let $\epsilon \in(0,1 / 6)$. For $c \in\left(0, \pi^{2} / 32\right)$, there exists $T_{c}$ such that, for all $T \geq T_{c}$,

$$
\mathbb{P}\left(\mathrm{R}_{\text {in }}(X)<T-T^{\frac{2}{3}+\epsilon}\right) \leq \exp \left\{-c T^{\frac{1}{3}+2 \epsilon}\right\} .
$$

For any constant $\hat{c}$ satisfying $\hat{c} \in\left(0, \pi^{2} / 2^{9}\right)$, there exists $T_{\hat{c}}$ such that, for all $T \geq T_{\hat{c}}$,

$$
\mathbb{P}\left(\mathrm{R}_{\text {out }}(X)>T+T^{\frac{2}{3}+\epsilon}\right) \leq \exp \left\{-\hat{c} T^{\frac{1}{3}+2 \epsilon}\right\} .
$$

How close is $X[0, T]$ to the boundary of its convex hull? This is a question about the local nature of the deviation of the conditioned process. We will write $\mathcal{L}(\operatorname{conv} K)$ for the length of the longest line segment that lies in the boundary of the convex hull $\operatorname{conv}(K)$ of $K$. We also define the maximum local roughness $\operatorname{MLR}(\mathrm{K})$ of $K$ to be the maximal distance between a point in $K$ and the boundary of $\operatorname{conv}(K)$. That is,

$$
\operatorname{MLR}(K):=\sup _{k \in K} \inf _{x \in \partial(\operatorname{conv}(K))} d(x, k) .
$$

We will write $\mathcal{L}(\operatorname{conv} B)$ for $\mathcal{L}(\operatorname{conv} K)$ in the case where $K$ is the range of the process $B:[0, T] \rightarrow \mathbb{R}^{2}$. A similar convention will apply for the maximum local roughness, and for the conditioned process $X:[0, T] \rightarrow \mathbb{R}^{2}$.

Senya Shlosman proposed this model to us, presenting some heuristic arguments that its deviation behaviour has much in common with that observed in numerous models of phase boundaries in two-dimensional random systems, more specifically, that exponents describing the typical behaviour of $\mathcal{L}(\operatorname{conv} X)$ and $\operatorname{MLR}(X)$ coincide with those in these other models. We will present some heuristic arguments of our own in favour of this belief at the end of the Introduction. Theorem 1 has the following straightforward consequence.

Corollary 1. The fluctuation of the conditioned process $X:[0, T] \rightarrow \mathbb{R}^{2}$ satisfies the following bounds. For any $\epsilon \in(0,1 / 12), c \in\left(0,2^{-21}\right)$, there exists $T_{c}$ such that, 
for all $T \geq T_{c}$,

$$
\mathbb{P}\left(\mathcal{L}(\operatorname{conv} X) \geq T^{\frac{5}{6}+\epsilon}\right) \leq \exp \left\{-c T^{\frac{1}{3}+4 \epsilon}\right\}
$$

Moreover, for $\epsilon \in(0,1 / 6)$, there exists $T_{\hat{c}}$ such that, for all $T \geq T_{\hat{c}}$,

$$
\mathbb{P}\left(\operatorname{arcl}(\partial(\operatorname{conv} X))>2 \pi\left(T+T^{\frac{2}{3}+\epsilon}\right)\right) \leq \exp \left\{-\hat{c} T^{\frac{1}{3}+2 \epsilon}\right\},
$$

where the constant $\hat{c}$ appears in Theorem 1, and where arcl denotes the arclength of a planar set.

Organisation. Section 2.1 describes more precisely the assertion from the theory of large deviations that the range $X[0, T]$ resembles a circle of radius $T$. The rest of Section 2 is devoted to providing the tools required for the proofs of the theorems (although we believe some of the results presented there are of independent interest). By considering the event that the motion $B:[0, T] \rightarrow \mathbb{R}^{2}$ encloses a given polygon of area $\pi T^{2}$, we provide in Section 2.2 a lower bound on the probability that $|\operatorname{enc}(B)| \geq$ $\pi T^{2}$, to which subsequent probabilities will be compared. The polygon whose vertices are given by the location of the motion $B$ at equally spaced time-points is introduced in Section 2.3 . The motion and the polygon are compared, notably in Lemma 4, where it is proved that the convex hull of the polygon is unlikely to trap significantly less area than that enclosed by the motion. The extent of the excess of area captured by the motion over that on which we condition is bounded above in Section 2.4. Proposition 2 of Section 2.5 proves that the conditioned motion is unlikely to significantly deviate from a constant velocity, in the sense of not covering distances of order $T^{2 / 3+2 \epsilon}$ in time of order $T^{2 / 3+\epsilon}$. The two major tools that we develop, Lemma 4 and Proposition 2, share an important element in common. In each case, we prove that the relevant event is unlikely by supplementing a bound arising from global information about the motion (essentially, that it encloses an area of $\pi T^{2}$ ) with a bound depending on the occurrence of another event, whose probability decays subexponentially. This latter event is independent of the first, and is a local feature of the motion: orthogonal fluctuations of the path from the sides of the polygon in the case of Lemma 4 and rapid motion along one side of an appropriately chosen polygon in the case of Proposition 2, We defer a further discussion of the ideas of the proof to the body of the paper.

Remark. Throughout, any time parameter takes a value in $[0, T]$. From time to time, terms such as $t+T^{2 / 3}$ appear that may be greater than $T$. In such cases, we are referring to the value on $[0, T]$ that is the value stated reduced modulo $T$.

1.1. Comparisons and heuristic arguments. It is believed that a variety of models of phase boundaries in two-dimensional random systems exhibit the same power-law fluctuations, even though their macroscopic profiles differ. An important example of such a model is that of a large finite cluster in the supercritical phase of the site percolation model in $\mathbb{Z}^{2}$. Choosing a parameter value $p>p_{c}$, Alexander and Uzun [7] condition on the event that there exists an open dual circuit surrounding the origin and enclosing an area of at least $n^{2}$, for large $n$. The asymptotic shape of this circuit is the boundary of a compact convex body, known as the Wulff crystal, that minimises a surface tension; cf. [10. The fluctuation of the circuit away from this shape may be measured by the maximum local roughness, which means in this case, the maximum distance of a vertex in the circuit from the boundary of its convex hull. In 11, the maximum local roughness is established to be bounded 
above by a quantity of the order of $n^{2 / 3}$. The average local roughness (which is, roughly speaking, the mean distance of a vertex in $C$ from the convex hull) is bounded above by $n^{1 / 3}$, up to a logarithmic correction. In [7, the maximum local roughness is shown to satisfy a lower bound that is given by $n^{1 / 3}$ if we omit logarthmic corrections.

We expect that the exponents describing the typical behaviour of the two measures of fluctuation, $\mathcal{L}$ and MLR, coincide with those anticipated for the percolation problem. That is, we expect that $\mathcal{L}(\operatorname{conv} X)$ behaves as $T^{2 / 3}$, and $\operatorname{MLR}(X)$ as $T^{1 / 3}$. To give an argument that supports the claim that $\mathcal{L}(\operatorname{conv} X)$ is typically not much greater than $T^{2 / 3}$, suppose that we sample the law of $X$ and find a realization where there is a line segment $L=\left[x_{1}, x_{2}\right]$ in $\partial(\operatorname{conv}(X))$ whose length exceeds $T^{2 / 3+\epsilon}$. The times $t_{1}$ and $t_{2}$ at which $X$ visits the endpoints $x_{1}$ and $x_{2}$ presumably satisfy $\left|t_{1}-t_{2}\right|>T^{2 / 3+\epsilon / 2}$, since the conditioned motion tends to move at a fairly constant rate. (Indeed, in Proposition 2, we will prove that the process $X$ is unlikely to cover distances as big as $L$ at speeds significantly greater than the average one at which $X$ moves.) Choosing two points $t$ and $t^{*}$ on the interval $[0, T]$ uniformly and independently of other randomness, we may resample the path of $X$ on the interval $\left[t, t^{*}\right]$, replacing $X\left[t, t^{*}\right]$ by a Brownian bridge that moves from $X(t)$ to $X\left(t^{*}\right)$ in time $t^{*}-t$. The Markov chain on loops that performs this resampling and jumps to the new path, provided that it captures the required area of $\pi T^{2}$ and stays put in the other case, has the law of the conditioned process $X$ as its invariant measure. We see that, for the action of this resampling on $X$, if the points $t$ and $t^{*}$ happen to be picked near to $t_{1}$ and $t_{2}$, respectively, then the effect of the resampling is to replace the motion of $X(w)$ as it traverses $L$ by a new motion. This motion has a time of at least $T^{2 / 3+\epsilon / 2}$ to traverse a distance of $T^{2 / 3+\epsilon}$. This new section of path typically fluctuates orthogonally to $L$ by a distance at least of order $T^{1 / 3+\epsilon / 4}$ (this being the square root of the available time). With a probability that is uniformly bounded below in $T$, this fluctuation occurs for a fixed but high fraction of time in the direction away from the existing convex hull of $X$. In this case, the resampled motion would seem to capture an area of the plane that exceeds that captured by $X(w)$ by an amount of the order of $T^{2 / 3+\epsilon} \cdot T^{1 / 3+\epsilon / 4}=T^{1+5 \epsilon / 4}$ (the left-hand side here being the product of the length of $L$ and the orthogonal fluctuation of the resampled motion). In this event, the resampling certainly meets the area criterion. Figure 1 1 shows a sketch of the range of a typical realization of $X$ and a resampling that creates more trapped area by the means just described. It is very believable that the typical order of the excess of area that $X$ captures over what it must capture, $|\operatorname{enc}(X)|-\pi T^{2}$, is linear in $T$, and that

$$
\mathbb{P}\left(|\operatorname{enc}(X)|-\pi T^{2} \geq T^{1+\alpha}\right)
$$

decays at a super-polynomial rate, for any given $\alpha>0$. However, the preceding argument suggests that the resampled motion - whose law is that of $X$ - has an excess of area of order of $T^{1+5 \epsilon / 4}$ with a probability that is at least a polynomially decaying multiple of the probability that there exists a line segment in $\partial(\operatorname{conv}(X))$ of length $T^{2 / 3+\epsilon}$ (the fact that the points $t$ and $t^{*}$ must be chosen to be near $t_{1}$ and $t_{2}$ is responsible for the appearance of a polynomial factor here). So, one expects that the probability of such a line segment in a realization of $X$ decays at a superpolynomial rate in $T$. It remains an interesting problem to derive such an upper bound on the quantity (1), as does that of obtaining lower bounds on the area 


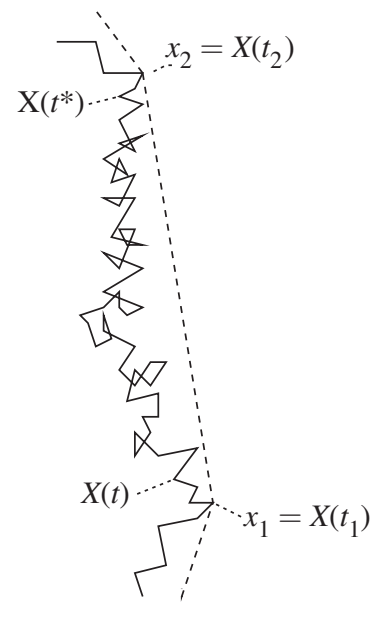

Before the resampling,

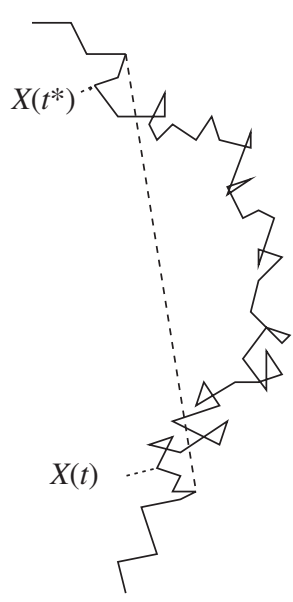

and afterwards.

Figure 2. How resampling may trap more area.

captured after resampling (an example of the difficulties involved in determining the area captured is the fact that Lemma 3 is not valid if the instance of $\operatorname{conv}(P)$ in its statement is replaced by $P$ ).

\section{UNDERSTANDING HOW THE PROCESS $X$ FLUCTUATES}

2.1. The macroscopic profile of the range of $X$. The theory of large deviations is of use in deriving the asymptotic shape of the range of the process $X$. Let $C_{0}\left([0,1], \mathbb{R}^{2}\right)$ denote the space of continuous planar-valued functions $f:[0,1] \rightarrow \mathbb{R}^{2}$ for which $f(0)=0$, and let $W$ denote two-dimensional Wiener measure. Writing $f_{T}(\cdot)=T f(\cdot / T)$, we have, by Theorem 5.1 of $[\underline{8}$,

$$
\limsup _{T \rightarrow \infty} \frac{1}{T} \log W\left\{g:\left.g\right|_{[0, T]}=f_{T}, \text { for some } f \in C\right\} \leq-\inf _{x \in C} I(x)
$$

for $C \subseteq C_{0}\left([0,1], \mathbb{R}^{2}\right)$ closed, and

$$
\liminf _{T \rightarrow \infty} \frac{1}{T} \log W\left\{g:\left.g\right|_{[0, T]}=f_{T}, \text { for some } f \in O\right\} \geq-\inf _{x \in O} I(x)
$$

for $O \subseteq C_{0}\left([0,1], \mathbb{R}^{2}\right)$ open.

The large deviations' rate function $I: C_{0}\left([0,1], \mathbb{R}^{2}\right) \rightarrow[0, \infty]$ is given by

$$
I(f)=\frac{1}{2} \int_{0}^{1}\|D f\|^{2}(t) d t
$$

if $f \in H_{1}\left([0,1], \mathbb{R}^{2}\right)$ (that is, if $f$ is absolutely continuous with square integrable derivative $D f)$, with $I(f)=\infty$ otherwise. By applying the contraction principle of large deviations [3, Section 4.2.1] to the mapping of the space of Brownian paths $Z:[0, T] \rightarrow \mathbb{R}^{2}$ to the space of Brownian loops $B:[0, T] \rightarrow \mathbb{R}^{2}$ given by $B(t)=Z(t)-\frac{t}{T} Z(T)$, we learn that (2) and (3) are valid for the measure $d B$ provided that the space $C_{0}\left([0,1], \mathbb{R}^{2}\right)$ is replaced by its subspace $\bar{C}_{0}$ consisting of 
functions $f$ for which $f(1)=0$. In evaluating the area enclosed by a loop, we will use for the present argument the signed area, given by

$$
A(f)=\frac{1}{2} \int_{0}^{s}\left(f_{1}(t) f_{2}^{\prime}(t)-f_{1}^{\prime}(t) f_{2}(t)\right) d t,
$$

for any $f \in H_{1}\left([0, s], \mathbb{R}^{2}\right)$ with $s>0$. Noting that $A\left(f_{T}\right) \geq \pi T^{2}$ for such $f$ if and only if $A(f) \geq \pi$, we now identify those functions in $\left\{f \in H_{1}\left([0,1], \mathbb{R}^{2}\right): A(f) \geq \pi\right\}$ that minimise $I(f)$. Any $f$ in this set is certainly square integrable, and thus, has an $L^{2}$-convergent complex Fourier series

$$
f(x)=\sum_{n \in \mathbb{Z}} a_{n} \exp \{2 n \pi i x\} .
$$

Note that, in these terms,

$$
I(f)=2 \pi^{2} \sum_{n \in \mathbb{Z}} n^{2}\left|a_{n}\right|^{2} .
$$

The formula (4) for signed area translates to

$$
A(f)=\pi \sum_{n \in \mathbb{Z}} n\left|a_{n}\right|^{2}=\pi \sum_{n=1}^{\infty} n\left(\left|a_{n}\right|^{2}-\left|a_{-n}\right|^{2}\right) .
$$

It is clear from (6) and (7) that any $f:[0,1] \rightarrow \mathbb{C}$ that minimises $I(f)$ among functions in $\bar{C}_{0}$ for which $A(f) \geq \pi$ has $a_{n}=0$ for $n<0$. It also follows from (6) and (77) that

$$
I(f) \geq 2 \pi A(f)
$$

for $f$ having only positive Fourier modes. Thus, we must have $I(f) \geq 2 \pi^{2}$ for functions $f$ such that $A(f) \geq \pi$. Note however that if $f(x)=-a+a \exp \{2 \pi i x\}$, for $a \in \mathbb{C}$ such that $|a|=1$, then $I(f)=2 \pi^{2}$ and $A(f)=\pi$, so that equality in (8) is attained for such functions $f$. Noting that, if $f$ has only positive Fourier modes and has some $a_{n} \neq 0$ for $n>1$, the inequality in (8) is strict. Then we have deduced that each of the minimising functions takes the form of a progression at constant rate along the circumference of a circle of radius 1 .

However, it appears that the fluctuation behaviour of the conditioned process may not be understood by a direct application of the techniques of large deviations. We now begin to develop the tools required for our study of this deviation.

2.2. Lower bound on $\mathbb{P}\left(|\operatorname{enc}(B)| \geq \pi T^{2}\right)$. In this section, we find a lower bound on the probability that the Brownian loop captures the required area. To this end, we estimate the probability that a regular polygon with an order of $T^{1 / 3}$ vertices and an area of $\pi T^{2}$ is enclosed by the motion $B$. The fact that a power of $1 / 3$ is optimal for this computation is of little surprise, given our belief about the true fluctuation of the model.

Lemma 1. Let $G_{m}=G_{m}(T)$ denote a regular polygon with $m$ vertices that contains an area equal to $\pi T^{2}$. Then, for any constant $C_{1}$ satisfying $C_{1}>4 / 3$, and for all $T \geq T_{C_{1}}$ sufficiently high,

$$
\begin{aligned}
& \mathbb{P}\left(\operatorname{enc}(B) \supset x+G_{\left\lfloor T^{\frac{1}{3}}\right\rfloor} \text { for some } x \in \mathbb{R}^{2}\right) \\
\geq & \exp \left\{-2 \pi^{2} T-C_{1} T^{\frac{1}{3}} \log T\right\} .
\end{aligned}
$$


Proof. Denote the successive vertices of $G_{m}(T)$ by

$$
\left\{y_{i}: i \in\{1, \ldots, m\}\right\}
$$

where we set $m=\left\lfloor T^{1 / 3}\right\rfloor$ and let $D_{i}$ denote the line segment whose endpoints are the centre of $G_{m}$ and $y_{i}$. Let $\phi_{i}$ denote the disk of radius one whose centre lies on the continuation of $D_{i}$ at distance two from $y_{i}$. Let $M_{i}$ denote the open half-plane, disjoint from $G_{m}(T)$, whose boundary contains the line segment $\left[y_{i}, y_{i+1}\right]$ (in the case where $i=m$, the line segment $\left[y_{m}, y_{1}\right]$ ). Let $q \in \mathbb{R}^{2}$ be such that $q \in-\phi_{1}$. For $i \in\{0, \ldots, m-2\}$, let $A_{i}$ denote the event that

$$
\begin{aligned}
B\left(\frac{i T}{m}\right) & \in q+\phi_{i+1}, \\
B\left(\frac{(i+1) T}{m}\right) & \in q+\phi_{i+2},
\end{aligned}
$$

and

$$
B(t) \in q+M_{i+1} \text { for } t \in\left[\frac{i T}{m}, \frac{(i+1) T}{m}\right] .
$$

In the case where $i=m-1$, we use the same definition, with $\phi_{1}$ replacing $\phi_{i+2}$ in its statement. Note that

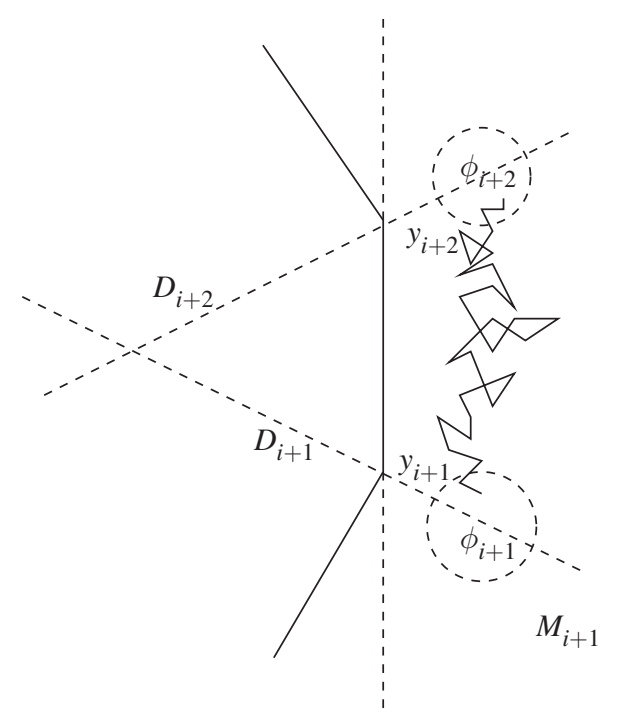

Figure 3. A realization of the event $A_{i}$.

$$
\bigcap_{i=0}^{m-1} A_{i} \subseteq\left\{\operatorname{enc}(B) \supset q+G_{m}\right\}
$$

We claim that

$$
\mathbb{P}\left(\bigcap_{i=1}^{m-1}\left\{B\left(\frac{i T}{m}\right) \in q+\phi_{i+1}\right\}\right) \geq \exp \left\{-2 \pi^{2} T-(2 / 3+o(1)) T^{\frac{1}{3}} \log T\right\}
$$


To see this, note that the left-hand side of (10) is given by

$$
\frac{m^{m}}{(2 \pi T)^{m-1}} \int \exp \left\{-\frac{m}{2 T} \sum_{i=0}^{m-1}\left\|x_{i+1}-x_{i}\right\|^{2}\right\} d x_{1} \ldots d x_{m-1}
$$

where the range of integration is equal to $\left(q+\phi_{2}\right) \times \ldots \times\left(q+\phi_{m}\right)$ and where we set $x_{0}=x_{m}=0$. The form for the expression in (11) occurs by computing the density of a finite-dimensional distribution of a Brownian bridge as the ratio of the corresponding density for a Brownian motion and the density at zero of a normal random variable with mean zero and variance $T$. It is straightforward to show that

$$
\left|D_{i}\right|=T+\frac{\pi^{2}}{3} T^{\frac{1}{3}}+O(1) .
$$

Thus, the distance between successive vertices satisfies

$$
\begin{aligned}
d\left(y_{i}, y_{i+1}\right) & =2\left|D_{i}\right| \sin \frac{\pi}{m} \leq \frac{2\left|D_{i}\right| \pi}{m} \\
& \leq \frac{2 \pi T}{m}+O(1) .
\end{aligned}
$$

From (13), it follows that the expression in (11) is bounded below by

$$
\frac{m^{m}}{(2 \pi T)^{m-1}}\left[\prod_{i=2}^{m} \operatorname{vol}\left(\phi_{i}\right)\right] \exp \left\{\frac{-m^{2}}{2 T}\left(\frac{2 \pi T}{m}+O(1)\right)^{2}\right\} \text {. }
$$

Since $\operatorname{vol}\left(\phi_{i}\right)=\pi$ for $i \in\left\{1, \ldots,\left\lfloor T^{1 / 3}\right\rfloor\right\}$, this expression is bounded below by $\exp \left\{-2 \pi^{2} T-(2 / 3+o(1)) T^{\frac{1}{3}} \log T\right\}$, as required to demonstrate that (10) holds. We claim that

$$
\begin{aligned}
& \mathbb{P}\left(\bigcap_{i=0}^{m-1} A_{i} \mid \bigcap_{i=1}^{m-1}\left\{B\left(\frac{i T}{m}\right) \in q+\phi_{i+1}\right\}\right) \\
\geq & \exp \left\{-\left(\frac{2}{3}+o(1)\right) T^{1 / 3} \log T\right\} .
\end{aligned}
$$

To see this, note that

$$
\begin{aligned}
& \mathbb{P}\left(\bigcap_{i=0}^{m-1} A_{i} \mid \bigcap_{i=1}^{m-1}\left\{B\left(\frac{i T}{m}\right) \in q+\phi_{i+1}\right\}\right) \\
= & \int \mathbb{P}\left(\bigcap_{i=0}^{m-1} A_{i} \mid \bigcap_{i=1}^{m-1}\left\{B\left(\frac{i T}{m}\right)=v_{i}\right\}\right) d \mu\left(v_{1}, \ldots, v_{m-1}\right),
\end{aligned}
$$

where $\mu$ is a probability measure on $\left(q+\phi_{2}\right) \times \ldots \times\left(q+\phi_{m}\right)$. Note further that, for any $\left(v_{1}, \ldots, v_{m-1}\right) \in\left(q+\phi_{2}\right) \times \ldots \times\left(q+\phi_{m}\right)$,

$$
\begin{aligned}
& \mathbb{P}\left(\bigcap_{i=0}^{m-1} A_{i} \mid \bigcap_{i=1}^{m-1}\left\{B\left(\frac{i T}{m}\right)=v_{i}\right\}\right) \\
= & \prod_{i=0}^{m-1} \mathbb{P}\left(A_{i} \mid\left\{B\left(\frac{i T}{m}\right)=v_{i}\right\} \cap\left\{B\left(\frac{(i+1) T}{m}\right)=v_{i+1}\right\}\right),
\end{aligned}
$$

where we set $v_{0}=v_{m}=0$. We condition on, for example,

$$
B\left(\frac{T}{m}\right)=v_{1}, B\left(\frac{2 T}{m}\right)=v_{2} .
$$


Consider the one-dimensional process $\hat{B}: I \rightarrow \mathbb{R}, I:=[T / m, 2 T / m]$, that is, the component of $B$ in the direction orthogonal to the line segment $[B(T / m)$, $B(2 T / m)$ ]. Under conditioning on (17), $\hat{B}$ is distributed as a Brownian bridge run to and from two given points, each of which we may insist lies in the interval $[-2,0]$. Note that the motion of $B$ will remain in the half-plane $M_{1}$ during the interval of time $I$ provided that $\hat{B} \leq 1 / 2$ throughout this time. The probability that the maximum of a one-dimensional Brownian bridge that is run for a given time starts at $a \in \mathbb{R}$ and ends at $b \in \mathbb{R}$, does not exceed a given value is a decreasing function of $a$ and of $b$. We may therefore assume that $\hat{B}(T / m)=0$ and $\hat{B}(2 T / m)=0$.

Recall that, if $M^{+}$denotes the maximum of the one-dimensional Brownian bridge run for time $T$, then, for any $r>0$,

$$
\mathbb{P}\left(M^{+}>r\right)=\exp \left\{-\frac{2 r^{2}}{T}\right\} .
$$

This assertion appears as formula (3.40) in [5, Chapter 4].

We deduce that

$$
\mathbb{P}(\hat{B}(t) \leq 1 / 2 \text { for all } t \in I) \geq 1-\exp \left\{-\frac{m}{2 T}\right\} \geq \frac{1}{8} T^{-\frac{2}{3}},
$$

the latter inequality being valid for high values of $T$.

Thus, given the occurrence of (17), the probability that the event $A_{1}$ occurs is at least $\frac{1}{8} T^{-2 / 3}$. From this, the product form of (16), and (15), follows (14).

From (10) and (14), we find that

$$
\mathbb{P}\left(\bigcap_{i=0}^{m-1} A_{i}\right) \geq \exp \left\{-2 \pi^{2} T-\left(\frac{4}{3}+o(1)\right) T^{\frac{1}{3}} \log T\right\} .
$$

The statement of the lemma follows from the inclusion (9).

2.3. The area captured by a polygonal approximation. The aim of this section is to prove Lemma 4 , which shows that it is unlikely that the motion $B$ captures the required area, while the convex hull of a certain approximating polygon fails to do so by a significant margin. Before turning to the proof, the following result is required.

Lemma 2. For any constant $C_{2} \geq 128 \pi$, the planar Brownian loop $B:[0, T] \rightarrow \mathbb{R}^{2}$ has the property that

$$
\sup _{t \in[0, T]}|B(t)|^{2}
$$

is bounded above in distribution by $C_{2} T+Z^{2}$, where $Z$ is a normal random variable with mean zero and variance $T$. That is, for all $a \in(0, \infty)$,

$$
\mathbb{P}\left(\sup _{t \in[0, T]}|B(t)|^{2} \geq a\right) \leq \mathbb{P}\left(C_{2} T+Z^{2} \geq a\right) .
$$

Remark. In fact, the method of proof we give would permit us to pick $Z$ to be distributed normally with mean zero and variance $(1 / 2+\epsilon) T$, for any $\epsilon>0$ (provided that the constant $C_{2}$ is changed suitably.) We made the choice $\epsilon=1 / 2$, because it is not valuable in the application to choose any lower value for $\epsilon$.

Proof. Note that

$$
\sup _{t \in[0, T]}|B(t)|^{2} \leq \sup _{t \in[0, T]} B_{1}(t)^{2}+\sup _{t \in[0, T]} B_{2}(t)^{2},
$$


where $B_{1}$ and $B_{2}$ denote the components of $B$. From the probability (18) that the maximum of a one-dimensional Brownian bridge exceeds a given level, and its counterpart for the minimum value reaching below a prescribed value, it follows that

$$
\mathbb{P}\left(\sup _{t \in[0, T]} B_{j}(t)^{2}>r^{2}\right) \leq 2 \exp \left\{-\frac{2 r^{2}}{T}\right\}, \text { for } j \in\{1,2\} .
$$

Replacing $r$ by $r / \sqrt{2}$ in (20), we obtain from (19) that

$$
\mathbb{P}\left(\sup _{t \in[0, T]}|B(t)|^{2}>r^{2}\right) \leq 4 \exp \left\{-\frac{r^{2}}{T}\right\}
$$

A lower bound on the tail of a random variable $Z$, distributed normally with mean zero and variance $T$, is now obtained:

$$
\begin{aligned}
\mathbb{P}\left(Z^{2}>r^{2}\right) & =\mathbb{P}\left(\frac{|Z|}{\sqrt{T}}>\frac{r}{\sqrt{T}}\right) \\
& \geq \frac{\frac{2 r}{\sqrt{T}}}{\left(\frac{r^{2}}{T}+1\right) \sqrt{2 \pi}} \exp \left\{-\frac{r^{2}}{2 T}\right\},
\end{aligned}
$$

where the inequality follows from a standard bound on the tail of the normal distribution, presented in Section 14.8 of [9. From (21) and (22), it follows that

$$
\mathbb{P}\left(\sup _{t \in[0, T]}|B(t)|^{2}>r^{2}\right) \leq \mathbb{P}\left(Z^{2}>r^{2}\right)
$$

for $r \geq C \sqrt{T}$, where $C=8 \sqrt{2 \pi}$. We have derived

$$
\mathbb{P}\left(\sup _{t \in[0, T]}|B(t)|^{2} \geq a\right) \leq \mathbb{P}\left(C_{2} T+Z^{2} \geq a\right)
$$

for any $C_{2} \geq 0$ and each $a \geq C^{2} T$. By choosing $C_{2}=C^{2}$, the right-hand side of (23) becomes equal to 1 for any $a \in\left[0, C^{2} T\right]$. This establishes the statement of the lemma.

Naturally enough, we seek to understand the behaviour of the conditioned motion $X$ by considering the polygon $P$ whose vertices are the locations of $X$ at $m$ equally spaced moments of time. As such, we are interested in bounding the discrepancy in the area captured by the range $X[0, T]$ and an approximating polygon. Some area is trapped by this range due to fluctuations of $X$ as it traverses between two adjacent vertices of $P$. Our analysis is complicated by the fact that $X[0, T]$ may also trap area in another non-local fashion, due to an overlap of the range of $X$ corresponding to sides of $P$ that are not adjacent. We introduce the relevant notation and then prove in Lemma 3 that the area captured by $X[0, T]$ is contained in the convex hull of $P$ up to local fluctuations.

Definition 1. Let $m \in \mathbb{N}$ and let $t^{\prime} \in[0, T]$.

- Let $P=P_{m}^{t^{\prime}}$ denote the polygon whose vertices are given by

$$
\left\{B\left(\frac{j T}{m}+t^{\prime}\right): j \in\{0, \ldots, m-1\}\right\} .
$$


- Let the length of the edges of $P_{m}^{t^{\prime}}$ be denoted by $\left\{L_{i}: i \in\{1, \ldots, m\}\right\}$, so that

$$
L_{i}=\left|B\left(\frac{i T}{m}+t^{\prime}\right)-B\left(\frac{(i-1) T}{m}+t^{\prime}\right)\right|,
$$

and let $l_{i}$ denote the line segment whose endpoints are $B\left((i-1) T / m+t^{\prime}\right)$ and $B\left(i T / m+t^{\prime}\right)$.

- For $i \in\{1, \ldots, m\}$, let $R_{i}=R_{i}\left(t^{\prime}\right)$ denote the maximum of the distance of the range of the motion $B$ from the segment $l_{i}$ during that interval of time in which $l_{i}$ is traversed by $B$. This is,

$$
R_{i}=\sup _{t \in[0, T / m]} d\left(B\left(\frac{(i-1) T}{m}+t^{\prime}+t\right), l_{i}\right),
$$

where $d$ denotes the distance between two sets in $\mathbb{R}^{2}$.

- For $i \in\{1, \ldots, m\}$, let $\hat{R}_{i}=\hat{R}_{i}\left(t^{\prime}\right)$ denote the maximum of the absolute value of the displacement of the motion from a point that traverses $l_{i}$ at a linear rate during the time $[(i-1) T / m, i T / m]$. That is,

$$
\begin{gathered}
\hat{R}_{i}=\sup _{t \in[0, T / m]} d\left(B\left(\frac{(i-1) T}{m}+t^{\prime}+t\right),\right. \\
\left.\left(1-\frac{m t}{T}\right) B\left(\frac{(i-1) T}{m}+t^{\prime}\right)+\frac{m t}{T} B\left(\frac{i T}{m}+t^{\prime}\right)\right) .
\end{gathered}
$$

- Let the set $Q_{i}=Q_{i}\left(t^{\prime}\right)$ be given by $Q_{i}=\left\{x \in \mathbb{R}^{2}: d\left(x, l_{i}\right) \leq R_{i}\right\}$.

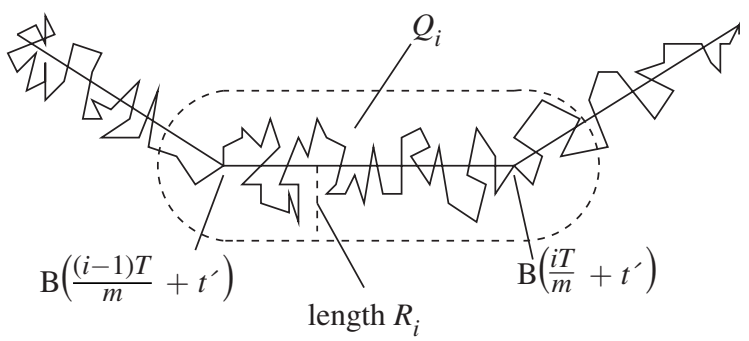

Figure 4. The objects of Definition 1.

Lemma 3. For any $T \in(0, \infty), t^{\prime} \in[0, T / m]$ and $m \in \mathbb{N}$,

$$
\operatorname{enc}(B) \subseteq \operatorname{conv}(P) \cup \bigcup_{i=1}^{m} Q_{i}
$$

Proof. The objects used in the proof are depicted in Figure 3. Take a point $y \in$ $\operatorname{enc}(B) \backslash \operatorname{conv}(P)$. Locate a half-plane $H$ that contains $\operatorname{conv}(P)$ and excludes $y$. Let $l^{\prime}$ denote the line through $y$ that intersects the boundary of $H$ at right angles. Note that there exists a point $z$ in the range of $B$ that lies on $l^{\prime}$ at a distance from the half-plane $H$ greater than that of $y$ : this is because $y \in \operatorname{enc}(B)$. Let $l_{i}$ be the interval in the polygon $P$ traversed while the Brownian path captures the point $z$; that is, $i \in\{1, \ldots, m\}$ is chosen so that

$$
z=B(t), \text { where } t \in\left[\frac{(i-1) T}{m}+t^{\prime}, \frac{i T}{m}+t^{\prime}\right] .
$$




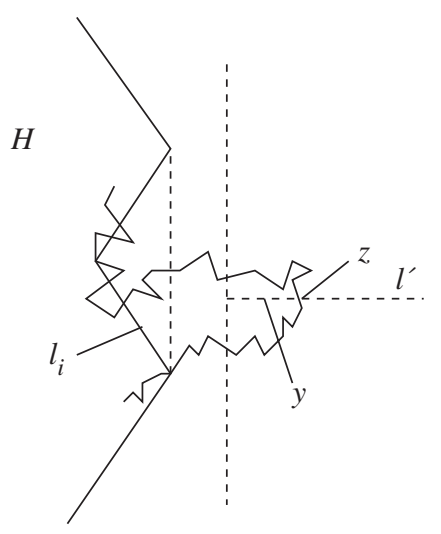

Figure 5. The proof of Lemma 3 ,

The point $y$ is closer to any given point in $H$ than is $z$, and each point in the line segment $l_{i}$ lies in $\operatorname{conv}(P)$, and so in $H$. Hence, $d\left(y, l_{i}\right) \leq d\left(z, l_{i}\right)$. That $d\left(z, l_{i}\right) \leq R_{i}$ follows from (25). Hence, $y \in Q_{i}$.

With these preliminaries in place, we will next state and prove Lemma 4. To outline the assertion and its proof, we are seeking to show that, given the occurrence of $|\operatorname{enc}(B)| \geq \pi T^{2}$, it is unlikely that the convex hull $\operatorname{conv}(P)$ of an approximating polygon with about $T^{1 / 3}$ sides has an area of order $\pi T^{2}-T^{4 / 3+\epsilon}$. We proceed as follows. If $|\operatorname{conv}(P)| \approx \pi T^{2}-T^{4 / 3+\epsilon}$, then the arclength of the polygon $P$ satisfies a lower bound of order $2 \pi T-\Theta\left(T^{1 / 3+\epsilon}\right)$. We regard this as a global first-order constraint. It has a probability that is slightly higher that the lower bound on $\mathbb{P}\left(|\operatorname{enc}(B)| \geq \pi T^{2}\right)$ provided by Lemma 1, (It is slightly more probable due to the shortfall of $\Theta\left(T^{1 / 3+\epsilon}\right)$ in arclength.) However, we learn from Lemma 3 that the $\Theta\left(T^{4 / 3+\epsilon}\right)$ shortfall in area trapped by $\partial(\operatorname{conv} P)$ compared to that captured by the motion $B$ lies in the union of fattenings $Q_{i}$ of the sides of $P$. Up to a small error, $\left|Q_{i}\right|$ is given by the product of the sidelength $L_{i}$ and the fluctuation $\hat{R}_{i}$ away from linear motion made by the process $X$ as it traverses the side. We obtain a lower bound on the sum (over sides, indexed by $i$ ) of the product $L_{i} \hat{R}_{i}$. The random variables $L_{i}$ and $\hat{R}_{i}$ being, in essence, normally distributed, we apply the Cauchy-Schwarz inequality to this sum and then use the law of the $\chi^{2}$ distribution to bound the probability that the sum $\sum_{i} L_{i} \hat{R}_{i}$ exceeds $\Theta\left(T^{4 / 3+\epsilon}\right)$.

The occurrence of this event, in addition to the global constraint on the arclength of $P$, forces the probability that both $|\operatorname{enc}(B)| \geq \pi T^{2}$ and $|\operatorname{conv}(P)| \approx \pi T^{2}-T^{4 / 3+\epsilon}$ occur below the estimate on $\mathbb{P}\left(|\operatorname{enc}(B)| \geq \pi T^{2}\right)$ provided by Lemma 1. In making use of this global effect and the presence of local distortion, it is crucial that the fluctuation $\hat{R}_{i}$ is independent of the sidelength $L_{i}$. Succinctly put, we find a means of extracting independent randomness from the fluctuations of the motion that run orthogonal to its overall direction, in the event whose conditional probability under $|\operatorname{enc}(B)| \geq \pi T^{2}$ we seek to show is small. As we mentioned at the end of the Introduction, this method is central to the approach of this paper. We will return to it, for example, in the proof of Proposition 2 . 
Lemma 4. Let $\epsilon \in[0,1 / 6)$, and let $m=\left\lfloor T^{1 / 3-\epsilon}\right\rfloor$. Suppose that the point $t^{\prime} \in[0, T]$ in the definition of the polygon $P$ is fixed, or that it is sampled randomly, according to an arbitrary distribution on $[0, T]$, and independently of the randomness that generates $B:[0, T] \rightarrow \mathbb{R}^{2}$. For $\epsilon^{\prime} \in(0,1 / 3)$, let $H=H\left(\epsilon^{\prime}\right)$ denote the event that

$$
|\operatorname{conv}(P)| \geq \pi T^{2}-T^{4 / 3+\epsilon^{\prime}},
$$

where $\operatorname{conv}(P)$ denotes the convex hull of the polygon P. Provided that $\epsilon^{\prime}>2 \epsilon$, for sufficiently large values of $T$,

$$
\mathbb{P}\left(H\left(\epsilon^{\prime}\right)^{c} \cap\left\{|\operatorname{enc}(B)| \geq \pi T^{2}\right\}\right) \leq \exp \left\{-2 \pi^{2} T-c_{1} T^{\frac{1}{3}+2\left(\epsilon^{\prime}-\epsilon\right)}\right\},
$$

where $c_{1}$ is any positive constant at most $\frac{2^{-10}}{108 \cdot(128)^{2} \pi^{4}}$.

Proof. For $a \in \mathbb{N}$, let $S_{a}$ denote the event that

$$
\pi T^{2}-2^{a} T^{4 / 3+\epsilon^{\prime}}>|\operatorname{conv}(P)| \geq \pi T^{2}-2^{a+1} T^{4 / 3+\epsilon^{\prime}},
$$

and let $R$ denote the event that the area of $\operatorname{conv}(P)$ is at most $(\pi-1 / 2) T^{2}$. Then

$$
H^{c}=\left(\bigcup_{a=0}^{k} S_{a}\right) \cup R,
$$

where $k=\left\lfloor\log _{2} T^{2 / 3-\epsilon^{\prime}}\right\rfloor-1$. We emphasize that, in general, this is not a disjoint union. Next, observe that

$$
\begin{aligned}
|\operatorname{enc}(B) \backslash \operatorname{conv}(P)| & \leq \sum_{i=1}^{m}\left|Q_{i}\right| \\
& \leq 2 \sum_{i=1}^{m}\left(L_{i}+2 R_{i}\right) R_{i} \\
& \leq 2 \sum_{i=1}^{m}\left(L_{i}+2 \hat{R}_{i}\right) \hat{R}_{i} \\
& \leq 2\left(\sum_{i=1}^{m} L_{i}^{2}\right)^{1 / 2}\left(\sum_{i=1}^{m} \hat{R}_{i}^{2}\right)^{1 / 2}+4 \sum_{i=1}^{m} \hat{R}_{i}^{2},
\end{aligned}
$$

where the first inequality follows from Lemma 3. The second inequality follows from the fact that $Q_{i}$ is contained in a rectangle of length $L_{i}+2 R_{i}$ and width $2 R_{i}$, while the third is implied by the inequality $R_{i} \leq \hat{R}_{i}$.

Let $a \in\{0, \ldots, k\}$. Note that, on the event $S_{a} \cap\left\{|\operatorname{enc}(B)| \geq \pi T^{2}\right\}$, we have that

$$
|\operatorname{enc}(B) \backslash \operatorname{conv}(P)| \geq|\operatorname{enc}(B)|-|\operatorname{conv}(P)| \geq 2^{a} T^{4 / 3+\epsilon^{\prime}} .
$$

We may write

$$
L_{i}^{2}=\frac{T\left(E_{2 i-1}^{2}+E_{2 i}^{2}\right)}{m},
$$

where, for each $i \in\{1, \ldots, m\}$, the quantities $\sqrt{T / m} E_{2 i-1}$ and $\sqrt{T / m} E_{2 i}$ are the horizontal and vertical components of the vector $B\left(i T / m+t^{\prime}\right)-B\left((i-1) T / m+t^{\prime}\right)$. 
As such, the family $\left\{E_{i}: i \in\{1, \ldots, 2 m\}\right\}$ is a collection of independent standard normal random variables, conditioned by insisting that

$$
\sum_{i=1}^{m} E_{2 i-1}=0, \sum_{i=1}^{m} E_{2 i}=0
$$

For each $i \in\{1, \ldots, m\}$ and any pair $(x, y) \in \mathbb{R}^{2}$, the conditional distribution of the random variable $\hat{R}_{i}$, given the event that $E_{2 i-1}=x$ and $E_{2 i}=y$, is independent of $x$ and $y$. Indeed, the process $Z_{i}:[(i-1) T / m, i T / m] \rightarrow \mathbb{R}^{2}$ given by

$$
Z_{i}(t)=B(t)-(1-(m t / T-(i-1))) B\left(\frac{(i-1) T}{m}\right)-(m t / T-(i-1)) B\left(\frac{i T}{m}\right)
$$

is a standard Brownian loop run for a time of $T / m$, no matter how we condition the values of the endpoints $B((i-1) T / m)$ and $B(i T / m)$. This means that, under any conditioning of the form $E_{2 i-1}=x$ and $E_{2 i}=y$, each $\hat{R}_{i}$ has the distribution of the maximal Euclidean distance of this Brownian loop. As such, we may apply Lemma 2 to find a collection of standard normal random variables $\left\{F_{i}: i \in\{1, \ldots, m\}\right\}$ for which

$$
\hat{R}_{i}^{2} \leq \frac{T}{m}\left(C_{2}+F_{i}^{2}\right) .
$$

The fact that the conditional distribution of $\hat{R}_{i}$ does not depend on $x$ and $y$ implies that we may assume that each $F_{i}$ is independent of $F_{j}$, for $j \in\{1, \ldots, m\}$ with $j \neq i$, and of each $E_{j}$, for $j \in\{1, \ldots, 2 m\}$.

From (27), (28), (29) and (31) there follows

$$
\frac{2 T}{m}\left(\sum_{i=1}^{2 m} E_{i}^{2}\right)^{1 / 2}\left(C_{2} m+\sum_{i=1}^{m} F_{i}^{2}\right)^{1 / 2}+4 C_{2} T+\frac{4 T}{m} \sum_{i=1}^{m} F_{i}^{2} \geq 2^{a} T^{4 / 3+\epsilon^{\prime}} .
$$

Using the inequalities $m \geq T^{1 / 3-\epsilon} / 2$ and $C_{2} \geq \sqrt{C_{2}} \geq 1$,

$$
\left(\sum_{i=1}^{2 m} E_{i}^{2}\right)^{1 / 2}\left(m+\sum_{i=1}^{m} F_{i}^{2}\right)^{1 / 2}+2 m+2 \sum_{i=1}^{m} F_{i}^{2} \geq \frac{2^{a-2} T^{2 / 3-\epsilon+\epsilon^{\prime}}}{C_{2}} .
$$

We note that

$$
\sum_{i=1}^{m} L_{i}^{2} \geq \frac{\left(\sum_{i=1}^{m} L_{i}\right)^{2}}{m} \geq \frac{(\operatorname{arcl}(\operatorname{conv}(P)))^{2}}{m} \geq \frac{4 \pi|\operatorname{conv}(P)|}{m},
$$

where arcl denotes arclength. The successive inequalities in (33) are consequences of the Cauchy-Schwarz inequality, the fact that the arclength of any polygon exceeds that of its convex hull, and the standard isoperimetric inequality, which in this case asserts that

$$
(\operatorname{arcl}(\operatorname{conv} P))^{2} \geq 4 \pi|\operatorname{conv}(P)|
$$

It follows from (29), (33) and the lower bound on $|\operatorname{conv}(P)|$ provided by the occurrence of the event $S_{a}$ that

$$
\sum_{i=1}^{2 m} E_{i}^{2} \geq 4 \pi^{2} T-2^{a+3} \pi T^{1 / 3+\epsilon^{\prime}}
$$


because the left- and right-hand side of this inequality are respectively bounded below and above by the quantity $\frac{4 \pi|\operatorname{conv}(P)|}{T}$. We see that the event

$$
\left\{|\operatorname{enc}(B)| \geq \pi T^{2}\right\} \cap S_{a}
$$

is contained in the event $F$, specified by the occurrence of the inequalities in (32) and (34). Note that we have the following inclusion:

$$
F \subseteq A_{C}^{1} \cup A_{C}^{2} \cup A_{C}^{3},
$$

where the events on the right-hand side are given by

$$
\begin{aligned}
A_{C}^{1}= & \left\{\sum_{i=1}^{2 m} E_{i}^{2} \geq C T\right\} \\
A_{C}^{2}= & \left\{\sum_{i=1}^{2 m} E_{i}^{2} \in\left[4 \pi^{2} T-2^{a+3} \pi T^{1 / 3+\epsilon^{\prime}}, C T\right)\right\} \\
& \cap\left\{m+\sum_{i=1}^{m} F_{i}^{2} \geq \sum_{i=1}^{2 m} E_{i}^{2}\right\}
\end{aligned}
$$

and

$$
\begin{aligned}
A_{C}^{3}= & \left\{\sum_{i=1}^{2 m} E_{i}^{2} \in\left[4 \pi^{2} T-2^{a+3} \pi T^{1 / 3+\epsilon^{\prime}}, C T\right)\right\} \\
& \cap\left\{m+\sum_{i=1}^{m} F_{i}^{2} \geq \frac{2^{2 a-4} T^{1 / 3-2 \epsilon+2 \epsilon^{\prime}}}{9 C C_{2}^{2}}\right\} .
\end{aligned}
$$

Here, $C$ denotes a fixed constant, and the constant $C_{2}$ satisfies the bound stated in Lemma 2. To derive (35), note that, if the event $F \cap\left(A_{C}^{1} \cup A_{C}^{2}\right)^{c}$ occurs, then

$$
\begin{aligned}
& 3\left(\sum_{i=1}^{2 m} E_{i}^{2}\right)^{\frac{1}{2}}\left(m+\sum_{i=1}^{m} F_{i}^{2}\right)^{\frac{1}{2}} \\
\geq & \left(\sum_{i=1}^{2 m} E_{i}^{2}\right)^{\frac{1}{2}}\left(m+\sum_{i=1}^{m} F_{i}^{2}\right)^{\frac{1}{2}}+2\left(m+\sum_{i=1}^{m} F_{i}^{2}\right) \\
\geq & \frac{2^{a-2}}{C_{2}} T^{\frac{2}{3}-\epsilon+\epsilon^{\prime}} .
\end{aligned}
$$

The first inequality follows from the fact that $m+\sum_{i=1}^{m} F_{i}^{2}<\sum_{i=1}^{2 m} E_{i}^{2}$, which holds on the event $F \cap\left(A_{C}^{1} \cup A_{C}^{2}\right)^{c}$, while the second is simply (32). Using (36), and the fact that $\sum_{i=1}^{2 m} E_{i}^{2}<C T$, we find that the second event of the two whose intersection defines $A_{C}^{3}$ must occur. The first occurs provided that both the event $\left(A_{C}^{1}\right)^{c}$ occurs and the inequality (34) is satisfied, which means that it occurs if the event $F \cap\left(A_{C}^{1} \cup A_{C}^{2}\right)^{c}$ occurs.

The standard normal random variables $\left\{E_{i}: i \in\{1, \ldots, 2 m\}\right\}$ are independent, conditioned only by the two linear constraints (30). Thus, the quantity $\sum_{i=1}^{2 m} E_{i}^{2}$ has the $\chi^{2}$-distribution with $2 m-2$ degrees of freedom. The random variable $\sum_{i=1}^{m} F_{i}^{2}$ has the $\chi^{2}$-distribution with $m$ degrees of freedom. Recall that the density 
$f_{m}:[0, \infty) \rightarrow[0, \infty)$ of this latter distribution is given by

$$
f_{m}(x)=\frac{x^{m / 2-1} \exp \{-x / 2\}}{2^{m / 2} \Gamma(m / 2)} .
$$

From this formula, we find that, for any given $C>0$ and all sufficiently high values of $T$,

$$
\mathbb{P}\left(A_{C}^{1}\right) \leq \exp \left\{-\frac{C T}{4}\right\}
$$

Recalling the independence property of the family of random variables $\left\{E_{i}: i \in\right.$ $\{1, \ldots, 2 m\}\}$ and $\left\{F_{i}: i \in\{1, \ldots, m\}\right\}$ asserted after (31), it follows that

$$
\mathbb{P}\left(A_{C}^{2}\right) \leq \int_{l}^{C T} f_{2 m-2}(x) d x \int_{l-m}^{\infty} f_{m}(x) d x
$$

where $l=4 \pi^{2} T-2^{a+3} \pi T^{1 / 3+\epsilon^{\prime}}$. The right-hand side of (39) may be bounded above by

$$
\begin{aligned}
& C T(C T)^{T^{1 / 3-\epsilon}} \exp \left\{-2 \pi^{2} T+2^{a+2} \pi T^{\frac{1}{3}+\epsilon^{\prime}}\right\} \\
& \times\left[C T(C T)^{T^{1 / 3-\epsilon}} \exp \left\{-2 \pi^{2} T+2^{a+2} \pi T^{\frac{1}{3}+\epsilon^{\prime}}+\frac{1}{2} T^{\frac{1}{3}-\epsilon}\right\}+\exp \{-C T / 4\}\right]
\end{aligned}
$$

by making use of $m \leq T^{1 / 3-\epsilon}$. From the fact that $2^{a+1} \leq T^{2 / 3-\epsilon^{\prime}}$ it follows that for any constant $c^{\prime}$ and for all $T$ exceeding a $c^{\prime}$-dependent constant,

$$
\mathbb{P}\left(A_{C}^{2}\right) \leq \exp \left\{-\left[4 \pi(\pi-1)-c^{\prime}\right] T\right\},
$$

provided that $C>8 \pi(\pi-1)$. On the event $A_{3}, \sum_{i=1}^{m} F_{i}^{2} \geq m$. This follows from the second requirement in the definition of $A_{3}$, along with the weaker than given inequality $\epsilon<2 \epsilon^{\prime}$, and $m \leq T^{1 / 3-\epsilon}$. Thus, if $A_{3}$ occurs, then

$$
\sum_{i=1}^{m} F_{i}^{2} \geq \frac{2^{2 a-5} T^{1 / 3-2 \epsilon+2 \epsilon^{\prime}}}{9 C C_{2}^{2}}
$$

Thus, by a similar estimate as in the case of $A_{C}^{2}$,

$$
\begin{aligned}
\mathbb{P}\left(A_{C}^{3}\right) \leq & C T(C T)^{T^{1 / 3-\epsilon}} \exp \left\{-2 \pi^{2} T+2^{a+2} \pi T^{1 / 3+\epsilon^{\prime}}\right\} \\
& \times\left[C T(C T)^{T^{1 / 3-\epsilon}} \exp \left\{-\frac{2^{2 a-6}}{9 C C_{2}^{2}} T^{\frac{1}{3}-2 \epsilon+2 \epsilon^{\prime}}\right\}+\exp \{-C T / 4\}\right] .
\end{aligned}
$$

Using the fact that $\epsilon^{\prime}>2 \epsilon$,

$$
\mathbb{P}\left(A_{C}^{3}\right) \leq \exp \left\{-2 \pi^{2} T-\frac{2^{2 a-7}}{9 C C_{2}^{2}} T^{\frac{1}{3}-2 \epsilon+2 \epsilon^{\prime}}\right\}+\exp \{-C T / 5\} .
$$

We find that

$$
\begin{aligned}
& \mathbb{P}\left(S_{a} \cap\left\{|\operatorname{enc}(B)| \geq \pi T^{2}\right\}\right) \\
\leq & \mathbb{P}\left(A_{C}^{1} \cup A_{C}^{2} \cup A_{C}^{3}\right) \leq \mathbb{P}\left(A_{C}^{1}\right)+\mathbb{P}\left(A_{C}^{2}\right)+\mathbb{P}\left(A_{C}^{3}\right) \\
\leq & \exp \left\{-2 \pi^{2} T-\frac{2^{2 a-8}}{9 C C_{2}^{2}} T^{\frac{1}{3}-2 \epsilon+2 \epsilon^{\prime}}\right\}+\exp \{-C T / 6\}
\end{aligned}
$$


where in the first inequality we used (35), and in the third, (38), (40) and (41). Recalling that $k=\left\lfloor\log _{2} T^{2 / 3-\epsilon^{\prime}}\right\rfloor-1$, we find that, provided $C>12 \pi^{2}$,

$$
\mathbb{P}\left(\left(\bigcup_{a=0}^{k} S_{a}\right) \cap\left\{|\operatorname{enc}(B)| \geq \pi T^{2}\right\}\right) \leq \exp \left\{-2 \pi^{2} T-c_{1} T^{\frac{1}{3}-2 \epsilon+2 \epsilon^{\prime}}\right\},
$$

for any constant $c_{1}$ satisfying

$$
c_{1} \in\left(0, \frac{2^{-9}}{9 C C_{2}^{2}}\right) \subseteq\left(0, \frac{2^{-10}}{9 \cdot 12 \cdot(128)^{2} \pi^{4}}\right),
$$

where the second inclusion is ensured by choosing $C_{2}=128 \pi$ and $C$ slightly above $12 \pi^{2}$.

By (26), it only remains to bound the probability of the event $R$ that appears there. Note that if $R$ occurs, then

$$
|\operatorname{enc}(B) \backslash \operatorname{conv}(P)| \geq \frac{1}{2} T^{2}
$$

From (27), we see that the occurrence of $R$ implies that

$$
2\left(\sum_{i=1}^{m} L_{i}^{2}\right)^{1 / 2}\left(\sum_{i=1}^{m} \hat{R}_{i}^{2}\right)^{1 / 2}+4 \sum_{i=1}^{m} \hat{R}_{i}^{2} \geq \frac{1}{2} T^{2}
$$

and so that

$$
\left(\sum_{i=1}^{2 m} E_{i}^{2}\right)^{1 / 2}\left(C_{2} m+\sum_{i=1}^{m} F_{i}^{2}\right)^{1 / 2}+2\left(C_{2} m+\sum_{i=1}^{m} F_{i}^{2}\right) \geq \frac{1}{8} T^{\frac{4}{3}-\epsilon},
$$

by use of (29), (31) and the inequality $m \geq \frac{1}{2} T^{1 / 3-\epsilon}$. If the inequality (42) is satisfied, then so is one of

$$
C_{2} m+\sum_{i=1}^{m} F_{i}^{2} \geq \frac{1}{24} T^{\frac{4}{3}-\epsilon}
$$

and

$$
\sum_{i=1}^{2 m} E_{i}^{2} \geq \frac{1}{24} T^{\frac{4}{3}-\epsilon}
$$

Since $m \leq T^{1 / 3}$ and $\epsilon<1 / 3$, each of these inequalitities is satisfied with a probability that decays at a rate faster than exponential. This completes the proof.

2.4. The excess of area. It is of interest to bound the excess of area captured by the motion, partly for the reasons presented in the heuristic discussion that ends the Introduction. In addition, we will make use of the following bound in the proof of Theorem 1 .

Proposition 1. For $\delta \in(0,1 / 3)$ and for all $T \in(0, \infty)$ sufficiently high, we have that

$$
\mathbb{P}\left(|\operatorname{enc}(B)| \geq \pi T^{2}+T^{\frac{4}{3}+\delta}\right) \leq \exp \left\{-2 \pi^{2} T-\frac{\pi}{4} T^{\frac{1}{3}+\delta}\right\} .
$$

Proof. We set $m=\left\lfloor T^{\frac{1}{3}}\right\rfloor$. Note that the event whose probability we seek to bound lies in $Y_{1} \cup Y_{2}$, where the events $Y_{1}$ and $Y_{2}$ are given by

$$
Y_{1}=\left\{|\operatorname{enc}(B)| \geq \pi T^{2}+T^{\frac{4}{3}+\delta}\right\} \cap\left\{|\operatorname{conv}(P)|<\pi T^{2}+\frac{1}{2} T^{\frac{4}{3}+\delta}\right\}
$$


and by

$$
Y_{2}=\left\{|\operatorname{conv}(P)| \geq \pi T^{2}+\frac{1}{2} T^{\frac{4}{3}+\delta}\right\} .
$$

Let $\hat{T} \in(0, \infty)$ be given by $\pi \hat{T}=\pi T+T^{\frac{1}{3}+\delta}$ and write $\hat{\mathbb{P}}$ for the law of a Brownian loop run for time $\hat{T}$. By Brownian scaling, we find that

$$
\begin{aligned}
\mathbb{P}\left(Y_{1}\right) & \leq \hat{\mathbb{P}}\left(\left\{|\operatorname{enc}(B)| \geq \pi \hat{T}^{2}\right\} \cap\left\{|\operatorname{conv}(P)|<\pi \hat{T}^{2}-\frac{1}{4} \hat{T}^{\frac{4}{3}+\delta}\right\}\right) \\
& \leq \exp \left\{-2 \pi^{2} T-\frac{c_{1}}{4^{2}} T^{\frac{1}{3}+2 \delta}\right\} .
\end{aligned}
$$

The first inequality in (43) follows for high values of $T$ from the fact that $\delta<2 / 3$. The second is an application of Lemma四 (with the choice $\epsilon^{\prime}=\delta-\frac{\log 4}{\log \hat{T}}$ ) and of the inequality $\hat{T} \geq T$.

Note that, by (29) and (33),

$$
\sum_{i=1}^{2 m} E_{i}^{2} \geq \frac{4 \pi}{T}|\operatorname{conv}(P)|,
$$

so that

$$
\mathbb{P}\left(Y_{2}\right) \leq \mathbb{P}\left(\sum_{i=1}^{2 m} E_{i}^{2} \geq 4 \pi^{2} T+2 \pi T^{\frac{1}{3}+\delta}\right) \leq \exp \left\{-2 \pi^{2} T-\frac{\pi}{2} T^{\frac{1}{3}+\delta}\right\} .
$$

We used the form (37) of the density of the $\chi^{2}$-distribution in the second inequality, as well as the fact that $m \leq T^{1 / 3}$. Applying the bounds (43) and (44) to estimate $\mathbb{P}\left(Y_{1} \cup Y_{2}\right)$, we deduce the statement of the proposition.

2.5. Regularity of the motion. We now present an estimate on the distance travelled by a planar Brownian loop during time periods of fixed duration.

Lemma 5. Given functions $f, g:[0, T] \rightarrow[0, \infty)$, let $Q_{f, g}$ denote the event that

$$
\sup _{t \in[0, T]} \sup _{s \in[t, t+f(T)]}|B(s)-B(t)| \leq g(T) .
$$

Provided that $f(T) \leq T$, we have that

$$
\mathbb{P}\left(Q_{f, g}^{c}\right) \leq\left(\frac{32 \sqrt{2} T}{\sqrt{\pi f(T)} g(T)}+\frac{16 \sqrt{T}}{\sqrt{\pi} g(T)}\right) \exp \left\{-\frac{g(T)^{2}}{128 f(T)}\right\} .
$$

Proof. First, note that

$$
Q_{f, g}^{c} \subseteq \bigcup_{j=1}^{\lfloor T / f(T)\rfloor} C_{j},
$$

where $C_{j}$ is the event that there exist $t_{1}, t_{2} \in[j f(T),(j+2) f(T)]$ for which $\left|B\left(t_{1}\right)-B\left(t_{2}\right)\right|>g(T)$. Noting that the process $B_{t}:[0, T] \rightarrow \mathbb{R}^{2}$ given by

$$
B_{t}(s)=B(t+s)-B(t)
$$

has the same law as $B=B_{0}$, we see that

(47) each of the events $C_{j}$ for $j \in\{1, \ldots,\lfloor T / f(T)\rfloor\}$ has an equal probability. 
We represent $B:[0, T] \rightarrow \mathbb{R}^{2}$ in the form $B(t)=W(t)-t W(T) / T$, where $W:$ $[0, T] \rightarrow \mathbb{R}^{2}$ is distributed as a standard planar Brownian motion. Note then that the inclusion

$$
C_{1} \subseteq\left(A_{1} \cap F^{c}\right) \cup F,
$$

where $A_{1}$ is the event that there exist $t_{1}, t_{2} \in[f(T), 3 f(T)]$ for which $\mid W\left(t_{1}\right)-$ $W\left(t_{2}\right) \mid>g(T) / 2$, and where $F$ is the event

$$
F=\left\{|W(T)|>\frac{T g(T)}{4 f(T)}\right\} .
$$

Indeed, on the event $C_{1} \cap F^{c}$, we find that

$$
\begin{aligned}
\left|W\left(t_{1}\right)-W\left(t_{2}\right)\right| & \geq\left|B\left(t_{1}\right)-B\left(t_{2}\right)\right|-\frac{\left|t_{1}-t_{2}\right|}{T}|W(T)| \\
& \geq g(T)-\left|t_{1}-t_{2}\right| \frac{g(T)}{4 f(T)} \geq \frac{1}{2} g(T),
\end{aligned}
$$

where $t_{1}, t_{2} \in[0, T]$ are as in the definition of the event $C_{j}$. Thus, $A_{j}$ occurs for that $j$ for which $t_{1}, t_{2} \in[j f(T),(j+2) f(T)]$. We have verified (48).

Given that, for each $j \in\{1, \ldots,\lfloor T / f(T)\rfloor\}$,

$$
\mathbb{P}\left(C_{j}\right)=\mathbb{P}\left(C_{1}\right) \leq \mathbb{P}\left(A_{1}\right)+\mathbb{P}(F),
$$

we seek to bound the probability of the events $A_{1}$ and $F$. To this end, note that, if $A_{1}$ occurs, then one of the components in the $x$ or $y$ direction of $W\left(t_{1}\right)-W\left(t_{2}\right)$ on $[f(T), 3 f(T)]$ has a difference between its maximum and minimum values that exceeds $g(T) /(2 \sqrt{2})$. For this component, one of the maximum and the absolute value of the minimum exceeds $g(T) /(4 \sqrt{2})$. Thus,

$$
\begin{aligned}
\mathbb{P}\left(A_{1}\right) & \leq 4 \mathbb{P}\left(\sup _{t \in[0,2 f(T)]} W_{1}(t)>\frac{g(T)}{4 \sqrt{2}}\right) \\
& =8 \mathbb{P}\left(W_{1}(2 f(T))>\frac{g(T)}{4 \sqrt{2}}\right) \\
& \leq \frac{32 \sqrt{2} \sqrt{f(T)}}{\sqrt{\pi} g(T)} \exp \left\{-\frac{g(T)^{2}}{128 f(T)}\right\},
\end{aligned}
$$

where in the equality, we used the reflection principle, and, in the latter inequality, a standard tail bound for a normal random variable (Theorem 1.4 of [4]).

To bound the probability of the event $F$, note that if $\frac{f(T)}{T}|W(T)|>\frac{1}{4} g(T)$, then at least one of the inequalities

$$
\frac{f(T)}{T}\left|W_{i}(T)\right|>\frac{1}{4 \sqrt{2}} g(T)
$$

holds for $i \in\{1,2\}$. By the same tail bound,

$$
\begin{aligned}
\mathbb{P}(F) & =\mathbb{P}\left(\frac{f(T)}{T}|W(T)|>\frac{1}{4} g(T)\right) \\
& \leq \frac{16 f(T)}{\sqrt{\pi T} g(T)} \exp \left\{-\frac{T g(T)^{2}}{64 f(T)^{2}}\right\} \leq \frac{16 f(T)}{\sqrt{\pi T} g(T)} \exp \left\{-\frac{g(T)^{2}}{64 f(T)}\right\}
\end{aligned}
$$

since $f(T) \leq T$. 
We now find, as required, that

$$
\begin{aligned}
\mathbb{P}\left(Q_{f, g}^{c}\right) & \leq \frac{T}{f(T)} \mathbb{P}\left(C_{1}\right) \leq \frac{T}{f(T)}\left(\mathbb{P}\left(A_{1}\right)+\mathbb{P}(F)\right) \\
& \leq\left(\frac{32 \sqrt{2} T}{\sqrt{\pi} \sqrt{f(T)} g(T)}+\frac{16 \sqrt{T}}{\sqrt{\pi} g(T)}\right) \exp \left\{-\frac{g(T)^{2}}{128 f(T)}\right\}
\end{aligned}
$$

by means of (46), (47) in the first inequality, (49) in the second, and (50) and (51) in the third.

Our next result provides an estimate on the regularity of the conditioned motion, to the effect that it is unlikely to move too quickly in short periods of time. More precisely, in a time of order $T^{2 / 3+\epsilon}$, we rarely see the motion cover as much distance as $T^{2 / 3+2 \epsilon}$. To summarise the proof, a lower bound on the sum of squares of sidelengths of an approximating polygon arises from the occurrence of the event $|\operatorname{enc}(B)| \geq \pi T^{2}$, because, by Lemma 4 , the convex hull of the polygon captures much the same area as the motion $B$ does, implying a lower bound on the arclength of the polygon. The condition that one side of the polygon be traversed by the motion unusually quickly means that, in the application of the Cauchy-Schwarz inequality by which the sum of squared sidelengths is compared to the arclength itself, the lower bound is increased over the case in which the vertices of the polygon are encountered by the motion $B$ at evenly spaced times. This extra factor, analogous to that arising from the lower bound on $\sum_{i} L_{i} \hat{R}_{i}$ in the proof of Lemma 4. forces the probability of the event that $B$ both traps an area of $\pi T^{2}$ and has a period of rapid motion below the lower bound on the probability of the event $|\operatorname{enc}(B)| \geq \pi T^{2}$ provided by Lemma 1 .

Proposition 2. For $\epsilon_{0} \in(0,1 / 3)$, let $\bar{J}_{\epsilon_{0}}$ denote the event that, for some $\epsilon \in$ $\left(\epsilon_{0}, 1 / 3\right)$ and for some $t \in[0, T]$,

$$
\left|B\left(t+T^{2 / 3+\epsilon}\right)-B(t)\right| \geq T^{\frac{2}{3}+2 \epsilon}
$$

Then

$$
\mathbb{P}\left(\bar{J}_{\epsilon_{0}} \cap\left\{|\operatorname{enc}(B)| \geq \pi T^{2}\right\}\right) \leq C_{3} T^{2} \log T \exp \left\{-2 \pi^{2} T-2^{-9} T^{\frac{2}{3}+3 \epsilon_{0}}\right\},
$$

where $C_{3}$ is any constant exceeding $256 \pi^{2}$.

We need a small lemma for the proof of Proposition 2 .

Lemma 6. For each $\epsilon_{0} \in(0,1 / 3)$ and $\delta \in\left(0, \epsilon_{0}\right)$, the occurrence of $\bar{J}_{\epsilon_{0}-\delta}^{c}$ implies that, for each $\epsilon>\epsilon_{0}$, if $t, t^{\prime} \in[0, T]$ satisfy $\left|B\left(t^{\prime}\right)-B(t)\right| \geq \frac{9}{2} T^{2 / 3+2 \epsilon}$, then $\left|t^{\prime}-t\right| \geq$ $\frac{1}{2} T^{2 / 3+\epsilon}$.

Proof. Given $\epsilon, t$ and $t^{\prime}$ satisfying the hypotheses of the lemma, we find that either

$$
\left|B\left(t+T^{\frac{2}{3}+\epsilon}\right)-B(t)\right| \geq \frac{9}{4} T^{\frac{2}{3}+2 \epsilon}
$$

or

$$
\left|B\left(t+T^{\frac{2}{3}+\epsilon}\right)-B\left(t^{\prime}\right)\right| \geq \frac{9}{4} T^{\frac{2}{3}+2 \epsilon} .
$$

If (52) applies, then certainly $\bar{J}_{\epsilon_{0}}$ occurs, since $\epsilon>\epsilon_{0}$. This means of course that $\bar{J}_{\epsilon_{0}-\delta}$ occurs as well. Supposing the second eventuality (53) and that $\left|t^{\prime}-t\right|<$ 
$\frac{1}{2} T^{2 / 3+\epsilon}$, we note that (53) may be rewritten as

$$
\left|B\left(t^{\prime}+T^{\frac{2}{3}+\epsilon^{\prime}}\right)-B\left(t^{\prime}\right)\right| \geq \frac{9}{4} T^{\frac{2}{3}+2 \epsilon},
$$

where the quantity $\epsilon^{\prime}$ is easily shown to satisfy the bounds

$$
\epsilon^{\prime} \in(\epsilon-\log 2 / \log T, \epsilon+\log (3 / 2) / \log T)
$$

and thus $\epsilon^{\prime}>\epsilon-\delta>\epsilon_{0}-\delta$, for high values of $T$. The right-hand side of (54) exceeds $T^{2 / 3+2 \epsilon^{\prime}}$ for high values of $T$, because (55) implies that $T^{2\left(\epsilon-\epsilon^{\prime}\right)} \geq 4 / 9$. We have shown that the occurrence of $\bar{J}_{\epsilon_{0}-\delta}$ is a consequence of (53) and the inequality $\left|t^{\prime}-t\right|<\frac{1}{2} T^{2 / 3+\epsilon}$. This establishes the statement of the lemma.

The proof of Proposition 2 depends largely on the following lemma.

Lemma 7. Let $\epsilon \in(0,1 / 3)$ and let $J=J(\epsilon)$ denote the event that, for some $t \in[0, T]$,

$$
\left|B\left(t+T^{2 / 3+\epsilon}\right)-B(t)\right| \geq \frac{1}{2} T^{\frac{2}{3}+2 \epsilon} .
$$

Then

$$
\mathbb{P}\left(J \cap\left\{|\operatorname{enc}(B)| \geq \pi T^{2}\right\}\right) \leq \exp \left\{-2 \pi^{2} T-2^{-9} T^{\frac{2}{3}+3 \epsilon}\right\} .
$$

Proof. Suppose that $\epsilon<1 / 5$ (the other case is simple, and will be handled at the end of the proof). Find $\epsilon^{\prime}>0$ satisfying $\epsilon^{\prime}<2 / 3, \epsilon^{\prime}-3 \epsilon<1 / 3$ and $5 \epsilon+1 / 3<2 \epsilon^{\prime}$ (these conditions may be satisfied, since $\epsilon<1 / 5$ ). We will consider the polygonal approximation of the range of $B$ in the case where $m=\left\lfloor T^{1 / 3-\epsilon}\right\rfloor$. In this context, the quantity $t^{\prime}$ of Definition 1 will be chosen to be a uniform random variable on the interval $[0, T / m]$ that is independent of the motion $B$. Note that

$$
H\left(\epsilon^{\prime}\right) \subseteq\left\{\sum_{i=1}^{m} L_{i} \geq 2 \pi T-\frac{\pi}{3} T^{\frac{1}{3}+\epsilon^{\prime}}\right\},
$$

where the event $H\left(\epsilon^{\prime}\right)$ was defined in the statment of Lemma 4 Indeed,

$$
\begin{aligned}
\sum_{i=1}^{m} L_{i} & \geq 2 \sqrt{\pi} \sqrt{|\operatorname{conv}(P)|} \\
& \geq 2 \pi \sqrt{T^{2}-\frac{T^{4 / 3+\epsilon^{\prime}}}{\pi}} \\
& \geq 2 \pi T-\frac{\pi}{3} T^{\frac{1}{3}+\epsilon^{\prime}},
\end{aligned}
$$

where the first inequality uses the bound displayed in (33). The second inequality is valid provided that $H\left(\epsilon^{\prime}\right)$ occurs, and the third is true for all $T$ sufficiently high, since $\epsilon^{\prime}<2 / 3$ and $3<\pi$.

For $a \geq 0$, let $K_{a}$ denote the event that

$$
2^{a-1} T^{\frac{2}{3}+2 \epsilon} \geq L_{1} \geq 2^{a-2} T^{\frac{2}{3}+2 \epsilon} .
$$

We claim that, for any $a \in \mathbb{N}$,

$$
H\left(\epsilon^{\prime}\right) \cap K_{a} \subseteq\left\{\sum_{i=1}^{2 m} E_{i}^{2} \geq 4 \pi^{2} T+2^{2 a-5} T^{\frac{2}{3}+3 \epsilon}\right\},
$$


where the collection of random variables $\left\{E_{i}: i \in\{1, \ldots, 2 m\}\right\}$ was introduced in (29). To derive (57), note that

$$
\sum_{i=1}^{m} L_{i}^{2} \geq L_{1}^{2}+\frac{\left(\sum_{i=2}^{m} L_{i}\right)^{2}}{m-1} .
$$

From (58) and (29) it follows that

$$
\sum_{i=1}^{2 m} E_{i}^{2} \geq \frac{m}{T} L_{1}^{2}+\frac{\left(\sum_{i=2}^{m} L_{i}\right)^{2}}{T} .
$$

Supposing the occurrence of the event $H\left(\epsilon^{\prime}\right) \cap K_{a}$, we find that, for high enough values of $T$,

$$
\begin{aligned}
\sum_{i=1}^{2 m} E_{i}^{2} & \geq \frac{T^{1 / 3-\epsilon}-1}{T} 2^{2 a-4} T^{\frac{4}{3}+4 \epsilon}+\frac{1}{T}\left(2 \pi T-\frac{\pi}{3} T^{\frac{1}{3}+\epsilon^{\prime}}-2^{a-1} T^{\frac{2}{3}+2 \epsilon}\right)^{2} \\
& \geq 2^{2 a-4} T^{\frac{2}{3}+3 \epsilon}-2^{2 a-4} T^{\frac{1}{3}+4 \epsilon}+4 \pi^{2} T-\frac{4 \pi^{2}}{3} T^{\frac{1}{3}+\epsilon^{\prime}}-2^{a+1} \pi T^{\frac{2}{3}+2 \epsilon} \\
& \geq 4 \pi^{2} T+2^{2 a-5} T^{\frac{2}{3}+3 \epsilon} .
\end{aligned}
$$

In the first inequality, (56) and (59) were used, while, in the third, the relations $\epsilon<1 / 3$ and $\epsilon^{\prime}-3 \epsilon<1 / 3$ were required.

From (57) and the definition of the random variables $E_{i}$ (with $i \in\{1, \ldots, 2 m\}$ ), it follows that

$$
\mathbb{P}\left(K_{a} \cap H\left(\epsilon^{\prime}\right)\right) \leq \int_{l}^{\infty} f_{2 m-2}(y) d y,
$$

where $f$ denotes the density of the $\chi^{2}$-distribution specified in (37), and the lower limit of integration $l$ is equal to $4 \pi^{2} T+2^{2 a-5} T^{\frac{2}{3}+3 \epsilon}$. Using the fact that $m \leq T^{\frac{1}{3}}$ in estimating the right-hand side of (60), we find that

$$
\mathbb{P}\left(K_{a} \cap H\left(\epsilon^{\prime}\right)\right) \leq \exp \left\{-2 \pi^{2} T-2^{2 a-7} T^{\frac{2}{3}+3 \epsilon}\right\}
$$

for all choices of $T$ exceeding a value that has no dependence on $a \geq 0$. An extra factor of one-half multiplies the term of $T^{\frac{2}{3}+3 \epsilon}$ in the last expression, in order to compensate for the non-leading terms in the expression for the $\chi^{2}$-density. By summing over $a \in \mathbb{N}$ in (61), we deduce that, for high values of $T$,

$$
\mathbb{P}\left(\left\{L_{1} \geq \frac{1}{4} T^{\frac{2}{3}+2 \epsilon}\right\} \cap H\left(\epsilon^{\prime}\right)\right) \leq \exp \left\{-2 \pi^{2} T-2^{-8} T^{\frac{2}{3}+3 \epsilon}\right\} .
$$

Allowing $I$ to denote the collection of those times $t \in[0, T]$ for which

$$
\left|B\left(t+T^{\frac{2}{3}+\epsilon}\right)-B(t)\right| \geq \frac{1}{2} T^{\frac{2}{3}+2 \epsilon},
$$

note that the set $I$ is distributionally invariant under shifts of $[0, T]$, and is nonempty if and only if the event $J(\epsilon)$ occurs. We denote by $I_{1}$ the event that there exists $t \in I$ satisfying $\left|t-t^{\prime}\right| \leq 1$, where the difference $t-t^{\prime}$ is being computed modulo $T$, as each other will be. We find that

$$
\mathbb{P}\left(I_{1} \cap\left\{|\operatorname{enc}(B)| \geq \pi T^{2}\right\} \mid J(\epsilon) \cap\left\{|\operatorname{enc}(B)| \geq \pi T^{2}\right\}\right) \geq \frac{2}{T} .
$$


If the event $I_{1}$ occurs, we have that

$$
\begin{aligned}
L_{1} & =\left|B\left(\frac{T}{m}+t^{\prime}\right)-B\left(t^{\prime}\right)\right| \\
& \geq\left|B\left(t+T^{\frac{2}{3}+\epsilon}\right)-B(t)\right|-\left|B\left(\frac{T}{m}+t^{\prime}\right)-B\left(t+T^{\frac{2}{3}+\epsilon}\right)\right|-\left|B\left(t^{\prime}\right)-B(t)\right|
\end{aligned}
$$

where $t \in I$ satisfies $\left|t-t^{\prime}\right| \leq 1$. Writing $f(T)=2 T^{\frac{1}{3}+2 \epsilon}+1$ and $g(T)=\frac{1}{8} T^{\frac{2}{3}+2 \epsilon}$, note that for high values of $T$, on the event $Q_{f, g}$,

$$
\left|B\left(\frac{T}{m}+t^{\prime}\right)-B\left(t+T^{\frac{2}{3}}\right)\right| \leq g(T) .
$$

This is because the difference between the two arguments of $B$ in (65) satisfies

$$
\begin{aligned}
\left|\left(\frac{T}{m}+t^{\prime}\right)-\left(t+T^{\frac{2}{3}}\right)\right| & \leq\left|\frac{T}{\left\lfloor T^{\frac{1}{3}-\epsilon}\right.}-T^{\frac{2}{3}+\epsilon}\right|+\left|t^{\prime}-t\right| \\
& \leq\left|\frac{T}{T^{\frac{1}{3}-\epsilon}-1}-T^{\frac{2}{3}+\epsilon}\right|+1 \\
& \leq T^{\frac{2}{3}+\epsilon} \sum_{j=1}^{\infty} T^{-j\left(\frac{1}{3}-\epsilon\right)}+1 \leq f(T)
\end{aligned}
$$

where the fact that $\epsilon \in(0,1 / 3)$ was used in the last inequality. Note also that, on the event $Q_{1, g}$,

$$
\left|B\left(t^{\prime}\right)-B(t)\right| \leq g(T)
$$

From (64), (65), (67) and the fact that $t \in I$, it follows that

$$
I_{1} \cap Q_{f, g} \cap Q_{1, g} \subseteq\left\{L_{1} \geq \frac{1}{4} T^{\frac{2}{3}+2 \epsilon}\right\}
$$

Thus,

$$
\begin{aligned}
& \mathbb{P}\left(\left\{L_{1} \geq \frac{1}{4} T^{\frac{2}{3}+2 \epsilon}\right\} \cap\left\{|\operatorname{enc}(B)| \geq \pi T^{2}\right\}\right) \\
\geq & \mathbb{P}\left(I_{1} \cap\left\{|\operatorname{enc}(B)| \geq \pi T^{2}\right\}\right)-\mathbb{P}\left(Q_{f, g}^{c}\right)-\mathbb{P}\left(Q_{1, g}^{c}\right) \\
\geq & \frac{2}{T} \mathbb{P}\left(J(\epsilon) \cap\left\{|\operatorname{enc}(B)| \geq \pi T^{2}\right\}\right) \\
& \quad C T \exp \left\{-c T^{1+2 \epsilon}\right\}-C T \exp \left\{-\frac{1}{64 \cdot 128} T^{\frac{4}{3}+4 \epsilon}\right\},
\end{aligned}
$$

with $c \in\left(0,128^{-2}\right)$. In the second inequality (63) was used, as well as the bound provided by Lemma 5. In applying this bound, we used the fact that $f, g \geq 1$ for 
$T$ sufficiently large. We find that

$$
\begin{aligned}
& \mathbb{P}\left(J(\epsilon) \cap\left\{|\operatorname{enc}(B)| \geq \pi T^{2}\right\}\right) \\
\leq & \frac{T}{2}\left[\mathbb{P}\left(\left\{L_{1} \geq \frac{1}{4} T^{\frac{2}{3}+2 \epsilon}\right\} \cap H\left(\epsilon^{\prime}\right)\right)+\mathbb{P}\left(H\left(\epsilon^{\prime}\right)^{c} \cap\left\{|\operatorname{enc}(B)| \geq \pi T^{2}\right\}\right)\right] \\
& \quad+C T^{2} \exp \left\{-c T^{1+2 \epsilon}\right\} \\
\leq & \frac{T}{2} \exp \left\{-2 \pi^{2} T-2^{-8} T^{\frac{2}{3}+3 \epsilon}\right\} \\
& +\frac{T}{2} \exp \left\{-2 \pi^{2} T-c_{1} T^{\frac{1}{3}+2\left(\epsilon^{\prime}-\epsilon\right)}\right\}+C T^{2} \exp \left\{-c T^{1+2 \epsilon}\right\},
\end{aligned}
$$

where (68) was used in the first inequality, the latter requiring (62) and an application of Lemma 4 (so that we use the inequality $\epsilon^{\prime}>2 \epsilon$, which follows from the assumption that $\left.2 \epsilon^{\prime}>5 \epsilon+1 / 3\right)$. From the fact that $5 \epsilon+1 / 3<2 \epsilon^{\prime}$ it follows that

$$
\mathbb{P}\left(J(\epsilon) \cap\left\{|\operatorname{enc}(B)| \geq \pi T^{2}\right\}\right) \leq \exp \left\{-2 \pi^{2} T-2^{-9} T^{\frac{2}{3}+3 \epsilon}\right\},
$$

for $T$ sufficiently high, as required.

There remains the case where $\epsilon \in[1 / 5,1 / 3)$. Note that, for any positive value for $\epsilon, J(\epsilon) \subseteq Q_{f, g}^{c}$, with the choices

$$
f(T)=T^{\frac{2}{3}+\epsilon}, g(T)=\frac{1}{4} T^{\frac{2}{3}+2 \epsilon}
$$

being made. We find that, in this case,

$$
\begin{aligned}
& \mathbb{P}\left(J(\epsilon) \cap\left\{|\operatorname{enc}(B)| \geq \pi T^{2}\right\}\right) \leq \mathbb{P}(J(\epsilon)) \leq \mathbb{P}\left(Q_{f, g}^{c}\right) \\
\leq & \frac{64}{\sqrt{\pi}}\left(2 \sqrt{2} T^{-\frac{5 \epsilon}{2}}+T^{-\frac{1}{6}-2 \epsilon}\right) \exp \left\{-\frac{1}{2048} T^{\frac{2}{3}+3 \epsilon}\right\},
\end{aligned}
$$

where Lemma 5 was applied in the third inequality. Given that $2 / 3+3 \epsilon>1$ for a choice of $\epsilon \in[1 / 5,1 / 3)$, we see that (69) establishes the statement of the lemma for such values of $\epsilon$.

Proof of Proposition 2. We claim that the following inclusion holds, for sufficiently high values of $T$ :

$$
\bigcup_{\epsilon^{\prime}}\left\{\exists t \in[0, T]:\left|B\left(t+T^{\frac{2}{3}+\epsilon^{\prime}}\right)-B(t)\right| \geq T^{\frac{2}{3}+2 \epsilon^{\prime}}\right\} \cap Q_{\frac{1}{C T}, 1} \subseteq J(\epsilon),
$$

where the union on the left-hand side is taken over values of $\epsilon^{\prime}$ satisfying $\left|\epsilon^{\prime}-\epsilon\right| \leq$ $\frac{T^{-5 / 3-\epsilon}}{2 C \log T}$. To derive (70), first set $V_{r}=\frac{T^{-5 / 3-r}}{2 C \log T}$ for $r>0$, and note that, for sufficiently high values of $T$, and for given $\epsilon>0$ and any $\epsilon^{\prime}$ satisfying

$$
\left|\epsilon^{\prime}-\epsilon\right| \leq V_{\epsilon}
$$

we have that

$$
\left|T^{\frac{2}{3}+\epsilon^{\prime}}-T^{\frac{2}{3}+\epsilon}\right| \leq \frac{1}{C T} .
$$

If the event on the left-hand side of (170) occurs, there exists $\epsilon^{\prime}$ satisfying $\left|\epsilon^{\prime}-\epsilon\right| \leq$ $T^{-5 / 3-\epsilon} /(2 C \log T)$ and $t \in[0, T]$ for which

$$
\left|B\left(t+T^{\frac{2}{3}+\epsilon^{\prime}}\right)-B(t)\right| \geq T^{\frac{2}{3}+2 \epsilon^{\prime}} .
$$


Note that, provided that the event on the left-hand side of (170) occurs,

$$
\begin{aligned}
& \left|B\left(t+T^{\frac{2}{3}+\epsilon}\right)-B(t)\right| \\
\geq & \left|B\left(t+T^{\frac{2}{3}+\epsilon^{\prime}}\right)-B(t)\right|-\left|B\left(t+T^{\frac{2}{3}+\epsilon^{\prime}}\right)-B\left(t+T^{\frac{2}{3}+\epsilon}\right)\right| \\
\geq & T^{\frac{2}{3}+2 \epsilon^{\prime}}-1=T^{\frac{2}{3}+2 \epsilon}+\left(T^{\frac{2}{3}+\epsilon^{\prime}}-T^{\frac{2}{3}+\epsilon}\right)\left(T^{\epsilon^{\prime}}+T^{\epsilon}\right)-1 \geq \frac{1}{2} T^{\frac{2}{3}+2 \epsilon},
\end{aligned}
$$

where the final inequality, valid for high values of $T$, is due to (71) and $\max \left\{\epsilon, \epsilon^{\prime}\right\}<$ 1. In the second inequality, we have used (71), (72) and the occurrence of $Q_{1 / C T, 1}$. This establishes the inclusion (70).

Note that

$$
\bar{J}_{\epsilon_{0}} \subseteq \bigcup_{i=1}^{N} \bigcup_{\epsilon^{\prime}:\left|\epsilon^{\prime}-\epsilon_{i}\right| \leq V_{\epsilon_{i}}}\left\{\exists t \in[0, T]:\left|B\left(t+T^{\frac{2}{3}+\epsilon^{\prime}}\right)-B(t)\right| \geq T^{\frac{2}{3}+2 \epsilon^{\prime}}\right\},
$$

where $\left\{\epsilon_{i}: i \in\{1, \ldots, N\}\right\}$ denotes a collection of values, each lying in $\left(\epsilon_{0}, 1 / 3\right)$, for which

$$
\left[\epsilon_{0}, \frac{1}{3}\right] \subseteq \bigcup_{i=1}^{N}\left(\epsilon_{i}-V_{\epsilon_{i}}, \epsilon_{i}+V_{\epsilon_{i}}\right) .
$$

As such, we may choose

$$
N \leq C T^{2} \log T
$$

From (70) and (73) it follows that

$$
\bar{J}_{\epsilon_{0}} \cap\left\{|\operatorname{enc}(B)| \geq \pi T^{2}\right\} \subseteq\left(\bigcup_{i=1}^{N} J\left(\epsilon_{i}\right) \cap\left\{|\operatorname{enc}(B)| \geq \pi T^{2}\right\}\right) \cup Q_{\frac{1}{C T}, 1}^{c} .
$$

From Lemma 7 the bound on $\mathbb{P}\left(Q_{f, g}\right)$ given by Lemma [5, and (74), it follows that

$$
\begin{aligned}
& \mathbb{P}\left(\bar{J}_{\epsilon_{0}} \cap\left\{|\operatorname{enc}(B)| \geq \pi T^{2}\right\}\right) \\
\leq & C T^{2} \log T \exp \left\{-2 \pi^{2} T-2^{-9} T^{\frac{2}{3}+3 \epsilon_{0}}\right\} \\
& +\left(\frac{32 \sqrt{2}}{\sqrt{\pi}} \sqrt{C} T^{\frac{3}{2}}+\frac{16}{\sqrt{\pi}} T^{\frac{1}{2}}\right) \exp \left\{-\frac{C}{128} T\right\} .
\end{aligned}
$$

Here the second term is negligible provided that $C>20 \pi^{2}$. Choosing $C_{3}=2 C$ yields the statement in the proposition.

\section{Proof of Theorem 1}

We now prove Theorem 1 pausing before doing so in order to explain the proof. We work with the approximating polygon $P$, with $m$ being chosen to be of the order $T^{1 / 3}$. The convex hull of $P$ is known typically to trap a high area by Lemma 4 . An isoperimetric result, Lemma 8 , gives a lower bound on the arclength of a planar convex body in terms of its area and its global deviation, the latter giving rise to an excess over that occurring in the extremal case of a disk. We deduce that the global deviation of the boundary of the convex hull of $P$ is not too high; for otherwise, the sum of the edge-lengths of the polygon being high implies that the $\chi^{2}$-distributed sum of their squares is improbably large. This arclength is also forced to be high if there is any vertex $p$ of $P$ that is too distant from the pertinent line segment 
in $\partial \operatorname{conv}(P)$. The fluctuation of the motion between two successive vertices of the polygon has been bounded in Proposition 2, We have obtained enough control on the motion to deduce that its global deviation typically satisfies an upper bound whose order is comparable to that satisfied by the deviation of the approximating polygon, that is, little more than $T^{2 / 3}$.

We so demonstrate that the motion is likely to be trapped between circles whose radii differ by an order that slightly exceeds that of $T^{2 / 3}$. The bounds on $\operatorname{MLR}(X)$ and $\mathcal{L}(X)$ given in Corollary 1 are straightforward consequences.

We now state the isoperimetric result that we require.

Lemma 8. Let $K$ denote a planar compact convex set whose area exceeds $\pi T^{2}$. Then

$$
\operatorname{arcl}(\partial K)^{2}>4 \pi^{2} T^{2}+\pi^{2}\left(\mathrm{R}_{\text {out }}(K)-\mathrm{R}_{\text {in }}(K)\right)^{2} .
$$

Proof. The result is implied by Bonnesen's inequality, as it is stated in the Theorem of Subsection 1.3.1, on page 3 of [2].

Proof of Theorem 1. We will prove that, for $\epsilon \in(0,1 / 6)$ and all sufficiently high $T$,

$$
\begin{aligned}
& \mathbb{P}\left(\left\{\mathrm{R}_{\text {out }}(B)-\mathrm{R}_{\text {in }}(B)>T^{\frac{2}{3}+\epsilon}\right\} \cap\left\{|\operatorname{enc}(B)| \geq \pi T^{2}\right\}\right) \\
\leq & \exp \left\{-2 \pi^{2} T-c_{4} T^{\frac{1}{3}+2 \epsilon}\right\},
\end{aligned}
$$

for any $c_{4} \in\left(0, \pi^{2} / 32\right)$. In doing so, we will make use of the polygon $P$, in the case where $m=\left\lfloor T^{\frac{1}{3}}\right\rfloor$ and $t^{\prime}=0$.

Definition 2. Let $\epsilon_{0}, \epsilon_{1}, \epsilon_{2}, \epsilon_{3}, \epsilon_{4}$ be positive constants that satisfy the following bounds: $\epsilon_{0}<1 / 3,2 \epsilon_{2}>\epsilon_{1}, \epsilon_{1}<1 / 6, \epsilon_{2}<1 / 3, \epsilon_{3}>\epsilon_{2} / 4, \epsilon_{4}>2 \epsilon_{0}$.

- Let $H_{1}$ denote the event that

$$
66 \pi^{3} T^{2} \geq|\operatorname{conv}(P)| \geq \pi T^{2}-T^{\frac{4}{3}+\epsilon_{1}} .
$$

- Let $H_{2}$ be given by

$$
H_{2}=\left\{\mathrm{R}_{\text {out }}(\operatorname{conv}(P))-\mathrm{R}_{\text {in }}(\operatorname{conv}(P)) \leq T^{\frac{2}{3}+\epsilon_{2}}\right\} .
$$

- Let $H_{3}$ be given by

$$
H_{3}=\left\{\sup _{k \in\{0, \ldots, m-1\}} d\left(\partial(\operatorname{conv} P), B\left(\frac{k T}{m}\right)\right) \leq T^{\frac{2}{3}+\epsilon_{3}}\right\} .
$$

- Let $H_{4}$ denote the event that, for each $i \in\{0, \ldots, m-1\}$,

$$
\sup _{t \in[0, T / m]}\left|B\left(\frac{(i-1) T}{m}+t\right)-B\left(\frac{(i-1) T}{m}\right)\right| \leq T^{\frac{2}{3}+\epsilon_{4}} .
$$

Note that

$$
\begin{aligned}
& \mathbb{P}\left(H_{1}^{c} \cap\left\{|\operatorname{enc}(B)| \geq \pi T^{2}\right\}\right) \\
& \leq \mathbb{P}\left(\left\{|\operatorname{conv}(P)|<\pi T^{2}-T^{\frac{4}{3}+\epsilon_{1}}\right\} \cap\left\{|\operatorname{enc}(B)| \geq \pi T^{2}\right\}\right) \\
&+\mathbb{P}\left(|\operatorname{conv}(P)|>66 \pi^{3} T^{2}\right) .
\end{aligned}
$$

The first event after the inequality in (76) coincides with $H\left(\epsilon_{1}\right)^{c} \cap\left\{|\operatorname{enc}(B)| \geq \pi T^{2}\right\}$ as it appears in Lemma 4, with $t^{\prime}$ set equal to zero. Applying this lemma with the 
choice $\epsilon=0$, we deduce that, for sufficiently high values of $T$, the first term on the right-hand side of (76) is bounded above by

$$
\exp \left\{-2 \pi^{2} T-c_{1} T^{\frac{1}{3}+2 \epsilon_{1}}\right\} \text {. }
$$

Note also that, if $|\operatorname{conv}(P)|>66 \pi^{3} T^{2}$, then

$$
\sup _{s, t \in[0, T]}|B(s)-B(t)|^{2} \geq \operatorname{diam}(\operatorname{conv}(P))^{2} \geq \frac{4}{\pi}|\operatorname{conv}(P)| \geq 4 \cdot 66 \pi^{2} T^{2},
$$

the first inequality being valid because the endpoints of the longest diameter of $\operatorname{conv}(P)$ are vertices $B\left(t_{1}\right)$ and $B\left(t_{2}\right)$ of $P$, and the second being the standard isoperimetric inequality. Note that (78) implies the occurrence of $Q_{T, T \pi \sqrt{8 \cdot 33}}^{c}$. Thus, we learn from Lemma 5 that

$$
\mathbb{P}\left(|\operatorname{conv}(P)|>66 \pi^{3} T^{2}\right) \leq \mathbb{P}\left(Q_{T, T \pi \sqrt{8 \cdot 33}}^{c}\right) \leq C \exp \left\{-\frac{33}{16} \pi^{2} T\right\} .
$$

By (76), the bound (77) on the first term on its right-hand side, and (79), we have that, for high values of $T$,

$$
\mathbb{P}\left(H_{1}^{c} \cap\left\{|\operatorname{enc}(B)| \geq \pi T^{2}\right\}\right) \leq \exp \left\{-2 \pi^{2} T-\frac{c_{1}}{2} T^{\frac{1}{3}+2 \epsilon_{1}}\right\} .
$$

We assert that

$$
\mathbb{P}\left(H_{1} \cap H_{2}^{c}\right) \leq \exp \left\{-2 \pi^{2} T-c_{2} T^{\frac{1}{3}+2 \epsilon_{2}}\right\},
$$

for any choice of $c_{2} \in\left(0, \pi^{2} / 2\right)$. To show this, we choose $\delta$ to satisfy $\delta<2 / 3$ and $\delta \in\left(\epsilon_{1}, 2 \epsilon_{2}\right)$. Note first that, since $\delta \in\left(\epsilon_{1}, 2 / 3\right)$,

$$
\pi T^{2}-T^{\frac{4}{3}+\epsilon_{1}} \geq \pi\left(T-T^{\frac{1}{3}+\delta}\right)^{2},
$$

for $T$ sufficiently high. The occurrence of $H_{1}$ therefore implies that

$$
|\operatorname{conv}(P)| \geq \pi\left(T-T^{\frac{1}{3}+\delta}\right)^{2},
$$

whereas, on the event $H_{2}^{c}$,

$$
\mathrm{R}_{\text {out }}(\operatorname{conv}(P))-\mathrm{R}_{\text {in }}(\operatorname{conv}(P))>T^{\frac{2}{3}+\epsilon_{2}} .
$$

Using the fact that $\delta<2 / 3$, we may apply Lemma 8 to find a lower bound on the arclength of the convex hull of the polygon $P$ in this eventuality:

$$
\operatorname{arcl}(\partial(\operatorname{conv} P))^{2} \geq 4 \pi^{2}\left(T-T^{\frac{1}{3}+\delta}\right)^{2}+\pi^{2} T^{\frac{4}{3}+2 \epsilon_{2}} .
$$

From (83) it follows that

$$
\begin{aligned}
\operatorname{arcl}(\partial(\operatorname{conv} P)) & \geq 2 \pi\left(T-T^{\frac{1}{3}+\delta}\right) \sqrt{1+\frac{1}{4} T^{\frac{-2}{3}+2 \epsilon_{2}}} \\
& \geq 2 \pi\left(T-T^{\frac{1}{3}+\delta}\right)\left(1+\left(\frac{1}{8}-o(1)\right) T^{\frac{-2}{3}+2 \epsilon_{2}}\right),
\end{aligned}
$$

where the facts that $\delta<2 / 3$ and $\epsilon_{2}<1 / 3$ were used in successive inequalities. Since $2 \epsilon_{2}>\delta$, we deduce that

$$
\operatorname{arcl}(\partial(\operatorname{conv} P)) \geq 2 \pi T+c T^{\frac{1}{3}+2 \epsilon_{2}}
$$


for any $c \in(0, \pi / 4)$. Recalling Definition 1, we find that

$$
\begin{aligned}
\sum_{i=1}^{m} L_{i}^{2} & \geq \frac{\left(\sum_{i=1}^{m} L_{i}\right)^{2}}{m} \geq \frac{(\operatorname{arcl}(\partial(\operatorname{conv} P)))^{2}}{m} \\
& \geq \frac{\left(2 \pi T+c T^{\frac{1}{3}+2 \epsilon_{2}}\right)^{2}}{m} \geq \frac{4 \pi^{2} T^{2}+4 \pi c T^{\frac{4}{3}+2 \epsilon_{2}}}{m} .
\end{aligned}
$$

Thus,

$$
\sum_{i=1}^{2 m} E_{i}^{2} \geq 4 \pi^{2} T+4 \pi c T^{\frac{1}{3}+2 \epsilon_{2}},
$$

where the collection of random variables $\left\{E_{i}: i \in\{1, \ldots, 2 m\}\right\}$ was introduced in (29). The left-hand side of (86) having the $\chi^{2}$-distribution with $2 m-2$ degrees of freedom, and $m$ being at most $T^{1 / 3}$, it follows by (37) that the probability of the occurrence of (86) is at most

$$
(C T)^{\frac{1}{2} T^{1 / 3}} \exp \left\{-2 \pi^{2} T-2 \pi c T^{1 / 3+2 \epsilon_{2}}\right\}+\exp \{-C T / 4\},
$$

for any constant $C$. Since $c$ may be chosen to lie arbitrarily close to $\pi / 4$, we have shown that (81) holds.

We will now show that

$$
\mathbb{P}\left(H_{1} \cap H_{2} \cap H_{3}^{c}\right) \leq \exp \left\{-2 \pi^{2} T-c_{3} T^{\frac{1}{2}}\right\},
$$

for any $c_{3} \in(0,2 \pi)$. We do so by proving the inclusion

$$
H_{1} \cap H_{2} \cap H_{3}^{c} \subseteq\left\{\sum_{i=1}^{m} L_{i}-\operatorname{arcl}(\partial(\operatorname{conv} P))>T^{\frac{1}{2}}\right\},
$$

for which purpose we require a lemma.

Lemma 9. Let $P$ denote a planar polygon, with vertex set $\left\{p_{i}: i \in\{1, \ldots, m\}\right\}$ (so that its arclength $\operatorname{arcl}(P)$ is given by the sum of its edge-lengths $\left|p_{i+1}-p_{i}\right|$ for $i \in\{1, \ldots, m\})$. Then

$$
\operatorname{arcl}(P) \geq \operatorname{arcl}(\partial(\operatorname{conv} P))+(\sqrt{5}-2) \min \left\{\frac{2 R^{2}}{Q}, R\right\},
$$

where $R=\sup _{i \in\{1, \ldots, m\}} d\left(p_{i}, \partial(\operatorname{conv} P)\right)$, and where $Q$ is equal to the supremum of the lengths of line segments in $\partial(\operatorname{conv} P)$.

Proof. Let $\mathrm{L}$ denote the collection of line segments that comprise $\partial(\operatorname{conv} P)$. Write $\partial_{\text {ext }}(P)$ for the exterior boundary of the polygon $P$. That is, $\partial_{\text {ext }}(P)=\partial\left(\operatorname{enc}(P)^{c}\right)$. Note that the set $\operatorname{conv}(P) \backslash \operatorname{enc}(P)$ is comprised of a finite number of connected components. The boundary of each component consists of the union of a line sement $l \in \mathrm{L}$ and a polygonal path lying in $\partial_{\text {ext }}(P)$ whose endpoints coincide with those of $l$. We denote this polygonal path by $l_{\text {ext }}$. In the case that $l \in \mathrm{L}$ does not arise from any such component, we set $l_{\text {ext }}=l$. Note that, for any pair $l^{\prime}, \tilde{l} \in \mathrm{L}$, the set $l_{\text {ext }}^{\prime} \cap \tilde{l}_{\text {ext }}$ has at most finitely many elements. Note further that $\left|l_{\text {ext }}\right| \geq|l|$ for each $l \in \mathrm{L}$, where we use $|\cdot|$ to denote the length of a line segment, or of a finite union of line segments. 
Let $k \in\{1, \ldots, m\}$ satisfy

$$
R=d\left(p_{k}, \partial(\operatorname{conv} P)\right) .
$$

We distinguish two cases, according to whether or not

$$
\left.d\left(p_{k}, \partial_{\text {ext }}(P)\right)\right) \leq \frac{R}{2} .
$$

Supposing that (90) holds, let $x \in \partial_{\text {ext }}(P)$ satisfy

$$
\left.d\left(x, p_{k}\right)\right) \leq \frac{R}{2}
$$

Let $l^{*}=\left[p_{i}, p_{j}\right]$ denote the element of $\mathrm{L}$ for which $x \in l_{\mathrm{ext}}^{*}$. By using (89) and (91), we find that

$$
d\left(x, l^{*}\right)>\frac{R}{2}
$$

Note also that

$$
\left|l_{\mathrm{ext}}^{*}\right| \geq\left|x-p_{i}\right|+\left|p_{j}-x\right| \geq\left|q-p_{i}\right|+\left|q-p_{j}\right|,
$$

where $q$ denotes the point at distance $\frac{R}{2}$ from $l^{*}$ whose projection onto this line segment is its midpoint. In the latter inequality, we invoked (92). Thus,

$$
\begin{aligned}
& \left|l_{\mathrm{ext}}^{*}\right| \geq \sqrt{\left|p_{j}-p_{i}\right|^{2}+R^{2}} \\
= & \left|p_{j}-p_{i}\right| \sqrt{1+\frac{R^{2}}{\left|p_{j}-p_{i}\right|^{2}}} .
\end{aligned}
$$

If

$$
\left|p_{j}-p_{i}\right|>2 R
$$

then

$$
\left|l_{\text {ext }}^{*}\right| \geq\left|p_{i}-p_{j}\right|+2(\sqrt{5}-2) \frac{R^{2}}{\left|p_{j}-p_{i}\right|} \geq\left|p_{i}-p_{j}\right|+2(\sqrt{5}-2) \frac{R^{2}}{Q} .
$$

In the first inequality of (96), we used (95) and the fact that

$$
\sqrt{1+x} \geq 1+2(\sqrt{5}-2) x
$$

for $x \in[0,1 / 4]$ to bound below the term appearing in (94). In the second inequality of (96), we used the fact that $\left|l^{*}\right| \leq Q$ (which follows from the definition of $Q$ ). Thus, provided that (90) and (95) hold, we have that

$$
\begin{aligned}
\operatorname{arcl}(P) & \geq \sum_{l \in \mathrm{L}}\left|l_{\text {ext }}\right| \geq \sum_{l \in \mathrm{L} \backslash\left\{l^{*}\right\}}|l|+\left|p_{i}-p_{j}\right|+2(\sqrt{5}-2) \frac{R^{2}}{Q} \\
& =\operatorname{arcl}(\partial(\operatorname{conv} P))+2(\sqrt{5}-2) \frac{R^{2}}{Q},
\end{aligned}
$$

where the second inequality is due to the fact that $\left|l_{\mathrm{ext}}\right| \geq|l|$ for each $l \in L$. Note that, if (95) fails, then

$$
\left|l_{\mathrm{ext}}^{*}\right|-\left|p_{i}-p_{j}\right| \geq \sqrt{\left|p_{i}-p_{j}\right|^{2}+R^{2}}-\left|p_{i}-p_{j}\right| \geq(\sqrt{5}-2) R,
$$


where the first inequality follows from (93) and where the second is obtained by minimising the value of the term $\sqrt{\left|p_{i}-p_{j}\right|^{2}+R^{2}}-\left|p_{i}-p_{j}\right|$ subject to the constraint that (95) does not hold. The inequality arising from (98) may replace (96) in deriving (97). We have established the statement of the lemma in the event that (90) holds. In the other case, there exists a union $\hat{l}$ of line segments in $P$ such that $p_{k} \in \hat{l},|\hat{l}| \geq \frac{R}{2}$, and $\hat{l} \cap \partial_{\text {ext }}(P)=\emptyset$. Given that $\left|\partial_{\text {ext }}(P)\right| \geq \operatorname{arcl}(\partial(\operatorname{conv} P))$ (which follows from $\left|l_{\text {ext }}\right| \geq|l|$ for each $\left.l \in \mathrm{L}\right)$, we find that

$$
\operatorname{arcl}(P) \geq\left|\partial_{\text {ext }}(P)\right|+|\hat{l}| \geq \operatorname{arcl}(\partial(\operatorname{conv} P))+\frac{R}{2} .
$$

Given that $\frac{1}{2}>\sqrt{5}-2$, this establishes the statement of the lemma in the case that (90) fails, thereby completing the proof of the lemma.

In applying Lemma 9, we first find an upper bound on the quantity $Q$ appearing in its statement, for the polygon $P$ under discussion. To be specific, we now show that, provided that $H_{1} \cap H_{2}$ occurs, then, for any line segment $L$ in the convex boundary of $P$,

$$
|L| \leq C_{4} T^{\frac{5}{6}+\frac{\epsilon_{2}}{2}}
$$

where $C_{4}$ is any constant exceeding $2^{3 / 2}(66)^{1 / 4} \sqrt{\pi}$. Indeed, if $H_{1} \cap H_{2}$ occurs, there exist two planar circles $J_{1}$ and $J_{2}$, satisfying

$$
\operatorname{rad}\left(J_{1}\right) \in\left[\frac{T}{2}, \pi \sqrt{66} T\right], \operatorname{rad}\left(J_{2}\right) \in\left[\operatorname{rad}\left(J_{1}\right), \operatorname{rad}\left(J_{1}\right)+T^{\frac{2}{3}+\epsilon_{2}}\right],
$$

and enc $\left(J_{1}\right) \subseteq \operatorname{enc}\left(J_{2}\right)$, such that $\partial(\operatorname{conv} P)$ lies in enc $\left(J_{2}\right) \backslash \operatorname{enc}\left(J_{1}\right)$.

The length of the longest line segment $L$ that lies in enc $\left(J_{2}\right) \backslash \operatorname{enc}\left(J_{1}\right)$ for a pair of circles $J_{1}$ and $J_{2}$ satisfying these conditions is attained when $\operatorname{rad}\left(J_{1}\right)$ and $\operatorname{rad}\left(J_{2}\right)$ are maximal, with the relative positions of $J_{1}, J_{2}$ and $L$ resembling the picture in Figure 6 . Thus, this length is bounded above by

$$
\begin{aligned}
& 2 \sqrt{\left(\pi \sqrt{66} T+T^{\frac{2}{3}+\epsilon_{2}}\right)^{2}-66 \pi^{2} T^{2}} \\
\leq & 2 \sqrt{2 \pi \sqrt{66} T^{\frac{5}{3}+\epsilon_{2}}+T^{\frac{4}{3}+2 \epsilon_{2}}} \leq 2^{3 / 2}(66)^{1 / 4} \sqrt{\pi}(1+o(1)) T^{\frac{5}{6}+\frac{\epsilon_{2}}{2}},
\end{aligned}
$$

the second inequality requiring $\epsilon_{2}<1 / 3$. This establishes (99).

To apply Lemma 9 and derive (88), note that the occurrence of $H_{3}^{c}$ implies that $R>T^{\frac{2}{3}+\epsilon_{3}}$. It follows from (99) that, in the event of $H_{1} \cap H_{2}$, we have the inequality $Q \leq C_{4} T^{\frac{5}{6}+\frac{\epsilon_{2}}{2}}$. Making use of the assumption that $\epsilon_{3}>\epsilon_{2} / 4$, we indeed obtain (88) from Lemma 9

Suppose that the quantity $\delta$ chosen before (82) satisfies $\delta<1 / 6$ (at this point, we require that $\epsilon_{1}<1 / 6$ ). Applying the standard isoperimetic inequality and using (82), which holds provided that $H_{1}$ occurs, we find that, on $H_{1}$,

$$
\operatorname{arcl}(\partial(\operatorname{conv} P)) \geq 2 \pi T-2 \pi T^{\frac{1}{3}+\delta} .
$$

From (97), whose validity we have established whether or not (95) holds, we deduce that

$$
\sum_{i=1}^{m} L_{i} \geq 2 \pi T+(1-o(1)) T^{\frac{1}{2}},
$$


since $\delta<1 / 6$. By a reprise of the argument that follows (85), the probability of this last inequality is at most $\exp \left\{-2 \pi^{2} T-2 \pi(1-o(1)) T^{\frac{1}{2}}\right\}$. We have now established (87).

Given that $\epsilon_{0}<\min \left\{1 / 3, \epsilon_{4} / 2\right\}$, Lemma 6 implies that $\bar{J}_{\epsilon_{0}}^{c} \subseteq H_{4}$. Let $\psi(T)=$ $T^{\frac{2}{3}}\left(T^{\epsilon_{2}}+2 T^{\epsilon_{3}}+2 T^{\epsilon_{4}}\right)$. It is straightforward that

$$
H_{2} \cap H_{3} \cap H_{4} \subseteq\left\{\mathrm{R}_{\text {out }}(B)-\mathrm{R}_{\text {in }}(B) \leq \psi(T)\right\} .
$$

From (101), it follows that

$$
\begin{aligned}
& \mathbb{P}\left(\left\{\mathrm{R}_{\text {out }}(B)-\mathrm{R}_{\text {in }}(B)>\psi(T)\right\} \cap\left\{|\operatorname{enc}(B)| \geq \pi T^{2}\right\}\right) \\
\leq & \mathbb{P}\left(\left(H_{2} \cap H_{3} \cap H_{4}\right)^{c} \cap\left\{|\operatorname{enc}(B)| \geq \pi T^{2}\right\}\right) \\
\leq & \mathbb{P}\left(H_{1}^{c} \cap\left\{|\operatorname{enc}(B)| \geq \pi T^{2}\right\}\right)+\mathbb{P}\left(H_{1} \cap H_{2}^{c}\right) \\
& +\mathbb{P}\left(H_{1} \cap H_{2} \cap H_{3}^{c}\right)+\mathbb{P}\left(\bar{J}_{\epsilon_{0}} \cap\left\{|\operatorname{enc}(B)| \geq \pi T^{2}\right\}\right) \\
(102) \leq & \exp \left\{-2 \pi^{2} T-\frac{c_{1}}{2} T^{\frac{1}{3}+2 \epsilon_{1}}\right\}+\exp \left\{-2 \pi^{2} T-c_{2} T^{\frac{1}{3}+2 \epsilon_{2}}\right\} \\
& +\exp \left\{-2 \pi^{2} T-c_{3} T^{\frac{1}{2}}\right\}+C_{3} T^{2} \log T \exp \left\{-2 \pi^{2} T-2^{-9} T^{\frac{2}{3}+3 \epsilon_{0}}\right\},
\end{aligned}
$$

where, in the second inequality, we used $H_{4}^{c} \subseteq \bar{J}_{\epsilon_{0}}$. The bounds (80), (81), (87) and that provided by Proposition 2 were used for the successive terms to obtain the third inequality. For $\epsilon \in(0,1 / 6)$, we set $\epsilon_{1}=\epsilon+\hat{\epsilon}, \epsilon_{2}=\epsilon_{4}=\epsilon, \epsilon_{3}=\epsilon / 4+\hat{\epsilon}$ and $\epsilon_{0}=\epsilon / 2-\hat{\epsilon}$, where $\hat{\epsilon}>0$ is chosen small enough that the restrictions on the parameters $\left\{\epsilon_{i}: i \in\{0, \ldots, 4\}\right\}$ are satisfied. We obtain

$$
\begin{aligned}
& \mathbb{P}\left(\left\{\mathrm{R}_{\text {out }}(B)-\mathrm{R}_{\text {in }}(B)>3 T^{\frac{2}{3}+\epsilon}\right\} \cap\left\{|\operatorname{enc}(B)| \geq \pi T^{2}\right\}\right) \\
\leq & \exp \left\{-2 \pi^{2} T-\left(c_{2}-o(1)\right) T^{\frac{1}{3}+2 \epsilon}\right\},
\end{aligned}
$$

since $\epsilon \in(0,1 / 6)$. From this follows (75) , the value of $c_{4}$ in (75) arising because we may choose $c_{2}$ in (81) to be slightly less than $\pi^{2} / 2$. Note that

$$
\begin{aligned}
& \left\{\mathrm{R}_{\text {in }}(B)<T-T^{\frac{2}{3}+\epsilon}\right\} \cap\left\{|\operatorname{enc}(B)| \geq \pi T^{2}\right\} \\
\subseteq & \left\{\mathrm{R}_{\text {out }}(B)-\mathrm{R}_{\text {in }}(B)>T^{\frac{2}{3}+\epsilon}\right\},
\end{aligned}
$$

because $\mathrm{R}_{\text {out }}(B) \geq T$ if $|\operatorname{enc}(B)| \geq \pi T^{2}$.

We obtain

$$
\begin{aligned}
& \mathbb{P}\left(\mathrm{R}_{\text {in }}(X)<T-T^{\frac{2}{3}+\epsilon}\right) \\
= & \frac{\mathbb{P}\left(\left\{\mathrm{R}_{\text {in }}(B)<T-T^{\frac{2}{3}+\epsilon}\right\} \cap\left\{|\operatorname{enc}(B)| \geq \pi T^{2}\right\}\right)}{\mathbb{P}\left(|\operatorname{enc}(B)| \geq \pi T^{2}\right)} \\
\leq & \exp \left\{-c_{4} T^{\frac{1}{3}+2 \epsilon}+C_{1} T^{\frac{1}{3}} \log T\right\},
\end{aligned}
$$

the inequality by means of (75), (103) and Lemma 1 . We have proved the first part of the theorem. 
To derive the second statement, note that

$$
\begin{aligned}
& \left\{\mathrm{R}_{\text {out }}(B)>T+2 T^{\frac{2}{3}+\epsilon}\right\} \cap\left\{|\operatorname{enc}(B)| \geq \pi T^{2}\right\} \\
\subseteq & \left\{\mathrm{R}_{\text {in }}(B)>T+T^{\frac{2}{3}+\epsilon}\right\} \\
& \cup\left\{\mathrm{R}_{\text {out }}(B)-\mathrm{R}_{\text {in }}(B)>T^{\frac{2}{3}+\epsilon}\right\} \cap\left\{|\operatorname{enc}(B)| \geq \pi T^{2}\right\} .
\end{aligned}
$$

Noting that the inequality $\mathrm{R}_{\text {in }}(B)>T+T^{\frac{2}{3}+\epsilon}$ implies that $|\operatorname{enc}(B)| \geq \pi T^{2}+$ $2 \pi T^{\frac{5}{3}+\epsilon}$, we may use Proposition 1 to bound the probability of the event in (104). We obtain that, for any $\alpha \in(0,1 / 3)$,

$$
\mathbb{P}\left(\mathrm{R}_{\mathrm{in}}(B)>T+T^{\frac{2}{3}+\epsilon}\right) \leq \exp \left\{-2 \pi^{2} T-T^{\frac{1}{3}+\alpha}\right\},
$$

provided that $T$ is chosen to be sufficiently high.

Using (75) to bound the probability of the event in (105), as well as (106), we find that, for $\epsilon \in(0,1 / 6)$ and for all sufficiently high values of $T$,

$$
\begin{aligned}
& \mathbb{P}\left(\left\{\mathrm{R}_{\text {out }}(B)>T+2 T^{\frac{2}{3}+\epsilon}\right\} \cap\left\{|\operatorname{enc}(B)| \geq \pi T^{2}\right\}\right) \\
\leq & \exp \left\{-2 \pi^{2} T-\frac{c_{4}}{2} T^{\frac{1}{3}+2 \epsilon}\right\} .
\end{aligned}
$$

From (107) and Lemma 1, we deduce the second statement of the theorem.

Lemma 10. Let $K$ denote a planar compact set. In the case that $\mathrm{R}_{\mathrm{in}}(K)>$ $\frac{1}{2} \mathrm{R}_{\text {out }}(K)$, we have that

$$
\mathcal{L}(\operatorname{conv} K) \leq 4 \sqrt{\mathrm{R}_{\text {in }}(K)\left(\mathrm{R}_{\text {out }}(K)-\mathrm{R}_{\text {in }}(K)\right)} .
$$

Proof. Given that $K$ is compact, we may locate circles $J_{1}$ and $J_{2}$ satisfying

$$
\begin{gathered}
\operatorname{enc}\left(J_{1}\right) \subseteq K \subseteq \operatorname{enc}\left(J_{2}\right), \operatorname{rad}\left(J_{2}\right)=\mathrm{R}_{\text {out }}(K) \\
\text { and } \operatorname{rad}\left(J_{1}\right)=\mathrm{R}_{\text {in }}(K) .
\end{gathered}
$$

Note that any line segment $L$ lying in $\partial(\operatorname{conv} K)$ satisfies

$$
L \subseteq \operatorname{int}\left(\operatorname{enc}\left(J_{1}\right)\right)^{c} \cap \operatorname{enc}\left(J_{2}\right),
$$

where $\operatorname{int}(A)$ denotes the interior of the set $A$. Indeed, if $\operatorname{int}\left(\operatorname{enc}\left(J_{1}\right)\right) \cap L \neq \emptyset$, there exists a point lying in enc $\left(J_{1}\right)$ and in the half-plane whose boundary contains $L$ and is disjoint from $\operatorname{conv}(K)$. This point does not lie in $K$, implying that enc $\left(J_{1}\right) \nsubseteq K$. The endpoints of $L$ lie in enc $\left(J_{2}\right)$, and thus, so does $L$, by the convexity of enc $\left(J_{2}\right)$.

Given that $\mathrm{R}_{\text {in }}(K)>\frac{1}{2} \mathrm{R}_{\text {out }}(K)$, the supremum of the lengths of line segments $L$ satisfying (109) over the set of pairs of circles $\left(J_{1}, J_{2}\right)$ satisfying (108) is achieved when $J_{1}$ and $J_{2}$ touch at a point, with $L$ being the line segment tangent to $J_{1}$ at the diametrically opposed point and having endpoints in $J_{2}$ (see Figure 6 ). For this choice of line segment, $|L|$ satisfies

$$
\begin{aligned}
|L| & =2 \sqrt{\mathrm{R}_{\text {out }}(K)^{2}-\left(2 \mathrm{R}_{\text {in }}(K)-\mathrm{R}_{\text {out }}(K)\right)^{2}} \\
& =4 \sqrt{\mathrm{R}_{\text {in }}(K)\left(\mathrm{R}_{\text {out }}(K)-\mathrm{R}_{\text {in }}(K)\right)}
\end{aligned}
$$

as required. 


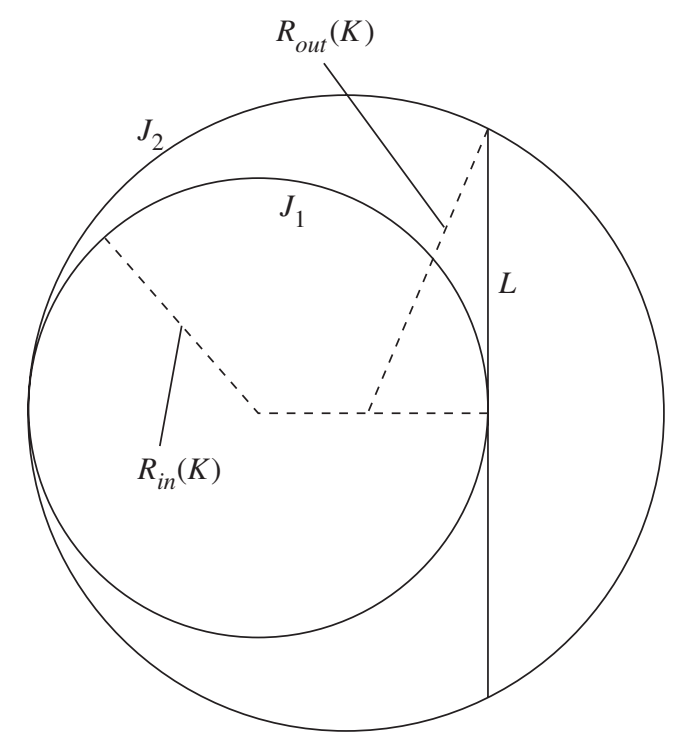

Figure 6. Computing the length $|L|$ in (110).

Proof of Corollary 1. From the first part of Lemma 10 follows the inclusion

$$
\begin{aligned}
& \left\{\mathcal{L}(\operatorname{conv} B)>T^{\frac{5}{6}+\epsilon}\right\} \\
\subseteq & \left\{\mathrm{R}_{\text {in }}(B)>C_{5} T\right\} \cup\left\{\mathrm{R}_{\text {out }}(B)-\mathrm{R}_{\text {in }}(B) \geq \frac{1}{16 C_{5}} T^{\frac{2}{3}+2 \epsilon}\right\},
\end{aligned}
$$

for any fixed $C_{5}>0$. Note that the event $Q_{f, g}^{c}$ occurs when the choices $f(T)=T$ and $g(T)=\mathrm{R}_{\text {in }}(B)$ are made. From Lemma 5, it follows that

$$
\mathbb{P}\left(\mathrm{R}_{\text {in }}(B)>C_{5} T\right) \leq \exp \left\{-\frac{C_{5}^{2}}{128} T\right\} .
$$

We deduce that

$$
\begin{aligned}
& \mathbb{P}\left(\left\{\mathcal{L}(\operatorname{conv} B)>T^{\frac{5}{6}+\epsilon}\right\} \cap\left\{|\operatorname{enc}(B)| \geq \pi T^{2}\right\}\right) \\
& \leq \mathbb{P}\left(\left\{\mathrm{R}_{\text {out }}(B)-\mathrm{R}_{\text {in }}(B)>\frac{1}{16 C_{5}} T^{\frac{2}{3}+2 \epsilon}\right\} \cap\left\{|\operatorname{enc}(B)| \geq \pi T^{2}\right\}\right) \\
& \quad+\mathbb{P}\left(\mathrm{R}_{\text {in }}(B)>C_{5} T\right) \\
& \leq \exp \left\{-2 \pi^{2} T-c_{5} T^{\frac{1}{3}+4 \epsilon}\right\}+C \exp \left\{-\frac{C_{5}^{2}}{128} T\right\},
\end{aligned}
$$

where $c_{5}$ is a constant satisfying $c_{5} \leq c_{4} /\left(16 C_{5}\right)^{2}$, and where $\epsilon \in(0,1 / 12)$. (Recall that the constant $c_{4}$ appeared in (75).) The first inequality is a consequence of (111). In the second, the bounds (112) and (75) were used. The choice $C_{5}>16 \pi$ ensures that the second term in (113) is negligible. Thus, $c_{5}$ may be chosen to be any value satisfying $c_{5} \leq 1 /\left(32(16)^{4}\right)=2^{-21}$. The first part of the corollary follows by applying Lemma 1 .

For the second part of the corollary, note that, if $\mathrm{R}_{\text {out }}(B) \leq T+T^{\frac{2}{3}+\epsilon}$, then $\operatorname{arcl}(\partial(\operatorname{conv} B)) \leq 2 \pi\left(T+T^{\frac{2}{3}+\epsilon}\right)$, because the arclength of the boundary of a planar 
convex set is monotone under containment [6]. Thus, the second part of Theorem 1 yields the result.

\section{ACKNOWLEDGMENTS}

The authors are grateful to Senya Shlosman for proposing the model to them, and thank him in particular for presenting heuristic arguments about the nature of its deviation. The authors thank Manjunath Krishnapur, Ben Hough and Dapeng Zhan for helpful comments on a draft version of the paper.

\section{REFERENCES}

[1] K. S. Alexander. Cube-root boundary fluctuations for droplets in random cluster models. Comm. Math. Phys., 224(3):733-781, 2001. MR.1871907 (2003i:82041)

[2] Yu. D. Burago and V. A. Zalgaller. Geometric inequalities, volume 285 of Grundlehren der Mathematischen Wissenschaften [Fundamental Principles of Mathematical Sciences]. Springer-Verlag, Berlin, 1988. Translated from the Russian by A. B. Sosinskiı̌, Springer Series in Soviet Mathematics. MR936419 (89b:52020)

[3] A. Dembo and O. Zeitouni. Large deviations and applications. In Handbook of stochastic analysis and applications, volume 163 of Statist. Textbooks Monogr., pages 361-416. Dekker, New York, 2002. MR.1882715

[4] R. Durrett. Probability: theory and examples. Duxbury Press, Belmont, CA, second edition, 1996. MR1609153 (98m:60001)

[5] I. Karatzas and S. E. Shreve. Brownian motion and stochastic calculus, volume 113 of Graduate Texts in Mathematics. Springer-Verlag, New York, 1988. MR917065 (89c:60096)

[6] L. A. Santaló. Integral geometry. In Studies in Global Geometry and Analysis, pages 147-193. Math. Assoc. Amer. (distributed by Prentice-Hall, Englewood Cliffs, N.J.), 1967. MR0215272 $(35: 6114)$

[7] H. B. Uzun and K. S. Alexander. Lower bounds for boundary roughness for droplets in Bernoulli percolation. Probab. Theory Related Fields, 127(1):62-88, 2003. MR2006231 (2004f:60209)

[8] S. R. S. Varadhan. Large deviations and applications, volume 46 of CBMS-NSF Regional Conference Series in Applied Mathematics. Society for Industrial and Applied Mathematics (SIAM), Philadelphia, PA, 1984. MR758258(86h:60067b)

[9] D. Williams. Probability with martingales. Cambridge Mathematical Textbooks. Cambridge University Press, Cambridge, 1991. MR.1155402 (93d:60002)

[10] G. Wulff. Zur Frage der Geschwingkeit des Wachstums und der Auflosung der Krystallflachen. Z. Kryst., 34:449-530, 1901.

Department of Mathematical Sciences, New York University-Courant Institute, 251 Mercer Street, New York, New York 10012-1185

Microsoft Research, One Microsoft Way, Redmond, Washington 98052 